\title{
Deformation Quantization of Heisenberg Manifolds
}

\author{
Marc A. Rieffel* \\ Department of Mathematics, University of California, Berkeley, CA 94720, USA
}

\begin{abstract}
For $M$ a smooth manifold equipped with a Poisson bracket, we formulate a $C^{*}$-algebra framework for deformation quantization, including the possibility of invariance under a Lie group of diffeomorphisms preserving the Poisson bracket. We then show that the much-studied non-commutative tori give examples of such deformation quantizations, invariant under the usual action of ordinary tori. Going beyond this, the main results of the paper provide a construction of invariant deformation quantizations for those Poisson brackets on Heisenberg manifolds which are invariant under the action of the Heisenberg Lie group, and for various generalizations suggested by this class of examples. Interesting examples are obtained of simple $C^{*}$-algebras on which the Heisenberg group acts ergodically.
\end{abstract}

About a decade ago a new approach to the quantization of classical mechanical systems was introduced by Vey [30], and Flato, Fronsdal, Lichnerowicz and coauthors $[3,12]$. Their approach involves viewing quantization as a deformation of structure, and goes roughly as follows. A classical mechanical system is given by its phase space, which is a $C^{\infty}$-manifold $M$, together with a symplectic structure which defines a Poisson bracket $\{$,$\} . To quantize this system, one selects a suitable$ algebra $A$ of $C^{\infty}$ functions on $M$ (functions of compact support, Schwartz functions, polynomials, to the extent these make sense), with the product being pointwise multiplication. One then deforms this product "in the direction of" the Poisson bracket. That is, if we denote the deformation parameter by "Planck's constant" $\hbar$, taking real values in some interval about 0 , then one tries to define a family $*_{h}$ of associative but not necessarily commutative products parametrized by $\hbar$, in such a way that for $f, g \in A$ one has

$$
f *_{n} g \rightarrow f g
$$

\footnotetext{
* This work was supported in part by National Science Foundation grant DMS 8601900
} 
and

$$
\left(f *_{h} g-g *_{h} f\right) / i \hbar \rightarrow\{f, g\}
$$

as $\hbar \rightarrow 0$. There is by now a quite substantial literature concerning this deformation quantization (see e.g. $[2,13]$ and the references they give), but almost all of it deals only with formal deformations, that is with deformations consisting of formal power-series in $\hbar$ with coefficients in $A$. Almost no discussion is given concerning convergence questions. There is good reason for this, in that the convergence questions appear very difficult in general, and it is even possible that requiring convergence may sometimes be too restrictive for the applications to physics which the authors have in mind.

The subject of deformation quantization was brought to my attention quite recently by Alan Weinstein, who, having looked through some of my recent work on non-commutative tori [27], pointed out to me that the non-commutative tori should be able to be considered to be deformation quantizations of ordinary tori for an appropriate Poisson structure, all in such a way that the convergence questions could be handled. The purpose of this paper is to formulate precisely what should be meant by a deformation quantization in the framework of $C^{*}$ algebras, so as to make precise Weinstein's observation, and then to go a step beyond the non-commutative tori by showing that for certain nice Poisson structures on Heisenberg manifolds, and on various related classes of manifolds, one can again carry out deformation quantization in such a way that the convergence questions can be satisfactorially handled. Here, by the Heisenberg manifolds we mean the quotients of the Heisenberg Lie group of $3 \times 3$ uppertriangular matrices with ones on the diagonal by its subgroups of matrices having integer entries.

There is a further aspect of deformation quantization which is of central interest. In much of the literature, the classical mechanical systems which are studied possess a Lie group of symmetries acting on the system, and one seeks deformations which are compatible with this Lie group action. This situation also holds for the noncommutative tori, where the ordinary torus acts as a group of symmetries. Now the Heisenberg Lie group acts on the Heisenberg manifolds, and in the present paper we will be primarily concerned with deformation quantizations of the Heisenberg manifolds which are compatible with this action. One reason for our interest in this aspect is that the action of an ordinary torus on a non-commutative torus provides the latter with a smooth structure making it a non-commutative differentiable manifold on which Connes showed $[7,8]$ how to extend the apparatus of differential geometry involving connections, curvature, Chern classes, etc. In the same way, the action of the Heisenberg Lie group on the various algebras occurring in the deformation quantizations of the Heisenberg manifolds provides another class of examples of non-commutative differentiable manifolds to which Connes' theory can be applied. This is also true of some of the other related examples we consider.

By way of contrast, we will show in the last section that the 2-sphere with its usual action of $S O(3)$ (or $(S U(2))$ does not admit deformation quantizations, of the strict kind studied here, which are compatible with the group action. This 
indicates that much remains to be clarified concerning exactly which systems do admit deformation quantizations of our strict kind.

Recently, Woronowicz [34-36,23] has obtained a kind of deformation of $S U(2)$ itself. This does not appear to fall precisely within our framework, since it is not clear whether his approach can be used to define deformed products on $C^{\infty}(S U(2))$, but it would be interesting to clarify exactly what the relationship is.

This paper is organized as follows. In the first section we define carefully our strict form of deformation quantization and show that the non-commutative tori indeed do provide examples, as suggested by Alan Weinstein. In the second section we introduce the Heisenberg manifolds and determine their invariant Poisson structures. These fall into two types which suggest generalizations in somewhat different directions. We study the first type of generalization in Sect. three, and the second type in Sects. four and five. In Sect. six we study briefly the structure of some of the $C^{*}$-algebras obtained, the main aim being to show that they are often simple. Finally, in Sect. seven we discuss the situation for $S O(3)$ acting on the sphere mentioned above.

We leave to a possible later paper the study of the non-commutative algebraic topology and differential geometry of the algebras in the deformation quantizations of the Heisenberg manifolds which we obtain, but our calculations so far support their being considered "non-commutative Heisenberg manifolds."

\section{Strict Deformation Quantization and Non-Commutative Tori}

Let $M$ be a $C^{\infty}$ manifold, and let $C^{\infty}(M)$ denote the associative algebra of $C^{\infty}$ complex-valued functions on $M$, with pointwise multiplication, and with involution given by complex conjugation $\left(f^{*}=\bar{f}\right)$. By a Poisson bracket on $M$ is meant a Lie algebra structure $\{$,$\} on the linear space C^{\infty}(M)$, such that for every $f \in C^{\infty}(M)$ the linear map $g \mapsto\{f, g\}$ from $C^{\infty}(M)$ to itself is a derivation of the associative algebra structure on $C^{\infty}(M)$. We also require that $\{$,$\} be real in the sense that$ $\left\{f^{*}, g^{*}\right\}=\{f, g\}^{*}$. Let $T M$ denote the tangent bundle of $M$. Then to give a Poisson structure on $M$ is the same as to give a skew 2-vector field $\Lambda$ on $M$, that is, a cross-section of $\Lambda^{2} T M$, such that if we set

$$
\{f, g\}=\langle\Lambda, d f \wedge d g\rangle,
$$

then $\{$,$\} satisfies the Jacobi identity (which is a quite stringent condition on \Lambda$ ).

If $M$ is compact, then for our present purposes $C^{\infty}(M)$ seems to be the only reasonable choice of algebra to consider for deformation quantization. But if $M$ is not compact, the situation is less clear. One evident candidate is $C_{c}^{\infty}(M)$, the algebra of functions of compact support. But in later sections we will be working extensively with the Heisenberg group, and with Fourier transforms, and in this setting the most convenient algebra will consist of the Schwartz functions. But we do not know how to define Schwartz functions on a general manifold. Another candidate is $C_{\infty}^{\infty}(M)$, the $C^{\infty}$ functions which vanish at infinity. In view of these various possibilities, our definition will be formulated in terms of any fixed *subalgebra, $A$, of $C_{\infty}^{\infty}(M)$ which contains $C_{c}^{\infty}(M)$ and is closed under taking Poisson brackets. Note then that $M$ is the maximal ideal space of $A$. 
1.1 Definition. Let $\Lambda$ and $A$ be as above. By a strict deformation quantization of $A$ in the direction of $\Lambda$, we will mean an open interval $I$ of real numbers containing 0 , together with, for each $\hbar \in I$, an associative product $*_{\hbar}$, an involution $*_{\hbar}$, and a $C^{*}$-norm \|\|$_{\hbar}$ (for $*_{\hbar}$ and $*_{\hbar}$ ) on $A$, which for $\hbar=0$ are the original pointwise product, complex conjugation involution, and supremum norm, such that

1. For every $f \in A$ the function $\hbar \mapsto\|f\|_{\hbar}$ is continuous

2. For every $f, g \in A,\left\|\left(f *_{h} g-g *_{\hbar} f\right) / i \hbar-\{f, g\}\right\|_{\hbar}$ converges to 0 as $\hbar$ goes to 0 .

We remark that if we let $A_{\hbar}$ denote the $C^{*}$-algebra obtained by completing $A$ for \|\|$_{h}$, then condition 1) is exactly what is needed to ensure that the family $\left\{A_{h}\right\}$, together with $A$ viewed as a *-algebra of cross-sections of this family, determines a continuous field of $C^{*}$-algebras. This follows from Proposition 10.2.3 and 10.3.2 of [9]. It is condition 2) which formalizes the idea that the deformation is "in the direction of $\Lambda$." Since this condition is essentially an infinitesimal condition at 0 , one does not expect strict deformation quantizations for a given $\Lambda$ to be unique, and indeed the case of the non-commutative tori considered later in this section already shows that uniqueness fails. This lack of uniqueness is one reason why questions of existence are difficult, since it shows that there will not be any canonical construction of strict deformation quantizations for a given $\Lambda$ (unless perhaps additional structure is imposed, such as perhaps a connection).

In order to make contact with the extensive literature concerning deformation quantization in terms of formal power series in $\hbar$, we would need to require that the above functions of $\hbar$ should actually be suitably analytic in $\hbar$. We will not explicitly need this condition, but it will, in fact, be true for many of the examples we will construct.

We remark that in most of the existing literature on deformation quantizations, no involution $*_{h}$ or norm \|\|$_{h}$ is considered. In fact, in some of the literature one of the objectives actually seems to be to get away from Hilbert spaces altogether.

We now indicate how to bring group actions into the picture.

1.2. Definition. Let $G$ be a Lie group, and let $\alpha$ be an action of $G$ as a group of diffeomorphisms of $\mathbf{M}$ which preserve the Poisson structure. Assume further that the corresponding action $\alpha$ of $G$ on $C^{\infty}(M)$ carries $A$ into itself. We will say that a strict deformation quantization of $A$, as defined above, is invariant under the action $\alpha$ if

1. For every $\hbar \in I$ and $x \in G$ the operator $\alpha_{x}$ on $A$ is an isometric $*$-automorphism for $*_{\hbar}, *_{\hbar}$, and \|\|$_{\hbar}$.

2. For every $f \in A$ and $\hbar \in I$ the map $x \mapsto \alpha_{x}(f)$ is a $C^{\infty}$ function on $G$, for the norm \|\|$_{\hbar}$.

3. There is an action, $\alpha$, of the Lie algebra $L$ of $G$ on $A$ which for each $\hbar \in I$ is by $*$-derivations of $A$ for $*_{\hbar}$ and $*_{\hbar}$, such that for $X \in L$ and $f \in A$

$$
\alpha_{X}(f)=\left.\frac{d}{d t}\right|_{t=0} \alpha_{\exp (t X)}(f)
$$

with respect to \|\|$_{\hbar}$. 
This definition will be adequate for our present purposes, but it has been shown by Arnal [1] that there are situations in which it is necessary to also allow the action $\alpha$ to vary as a function of $\hbar$. Even more, the example studied in [23] suggests that at times it will be necessary to perform a deformation quantization of the acting Lie group as well as of the Poisson manifold.

We now show that the non-commutative tori [27] are strict deformation quantizations of ordinary tori, thus making precise Alan Weinstein's observation to me that this should be the case. We let $T^{d}$ denote an ordinary $d$-dimensional torus, viewed as $R^{d} / Z^{d}$, and let $A=C^{\infty}\left(T^{d}\right)$. We let $G$ be $R^{d}$ (or $T^{d}$ ), acting on $A$ by translation in the evident way, with corresponding action of the Lie algebra $L \cong R^{d}$. For $i=1, \ldots, d$ let $X_{i}$ denote the vector field on $T^{d}$ corresponding to differentiation in the $i^{\text {th }}$ direction. Then it is easily seen that the $G$-invariant Poisson structures on $T^{d}$ are of the form

$$
\Lambda=-\pi^{-1} \sum_{j<k} \theta_{j k} X_{j} \wedge X_{k}
$$

where $\left\{\theta_{j k}\right\}$ is a real skew-symmetric matrix, and the factor $-\pi^{-1}$ is included so as to simplify later formulas. When convenient, we will also view $\theta$ as a skew bilinear form on $R^{d}$ or $Z^{d}$. We let $\tau$ be the Lebesgue measure on $T^{d}$ of total mass 1.

To obtain a corresponding strict deformation quantization, we now take the Fourier transform of the above set-up, much as one does to obtain the Moyal product [3] which is the deformation quantization for $M=R^{2 n}$ with its standard Poisson structure. The conventions for the Fourier transform which we now introduce to do this will also be used in later sections. We let $e$ be the function on $R$ defined by $e(t)=\exp (2 \pi i t)$. We view $T$ as $R / Z$, and for $f \in C^{\infty}\left(T^{d}\right)$ we define its Fourier transform, $\hat{f}$, on $Z^{d}$ by

$$
\hat{f}(n)=\int_{T^{d}} \bar{e}(n \cdot x) f(x) d \tau(x),
$$

where $n \cdot x$ denotes the standard inner product on $R^{d}$. It is well known that the Fourier transform carries $C^{\infty}\left(T^{d}\right)$ onto $S\left(Z^{d}\right)$ (the Schwartz space of those functions on $Z^{d}$ which go to zero at infinity faster than any polynomial grows). Furthermore, the pointwise product is carried to convolution, the involution is carried to $\hat{f}^{*}(n)=$ $\bar{f}(-n)$, Lebesgue measure $\tau$ is carried to evaluation at 0 , and translation by $c \in R^{d}$ is carried to pointwise multiplication by $n \mapsto e(n \cdot c)$. Then the operator $X_{j}$ corresponds to pointwise multiplication by $2 \pi i n_{j}$, and so the Poisson bracket is given, for $\phi, \psi \in S\left(Z^{d}\right)$ and $n \in Z^{d}$, by

$$
\begin{aligned}
\{\phi, \psi\}(n) & =4 \pi \sum_{m \in Z^{d}} \sum_{j, k} \theta_{j k} m_{j} \phi(m)\left(n_{k}-m_{k}\right) \psi(n-m) \\
& =4 \pi \sum \phi(m) \psi(n-m) \theta(m, n),
\end{aligned}
$$

since, for $\theta$ viewed as a skew form, $\theta(m, m)=0$.

Accordingly, as suggested by the Moyal product [3], we let the $I$ of Definition 1.1 be the whole real line $R$, and for any $\hbar \in I$ we define a bicharacter $\sigma_{\hbar}$ on $Z^{d}$ by

$$
\sigma_{\hbar}(m, n)=e(\hbar \theta(m, n)) \text {. }
$$


We then set

$$
\left(\phi *_{\hbar} \psi\right)(n)=\sum_{m} \phi(m) \psi(n-m) \sigma_{\hbar}(m, n) .
$$

Since $\sigma_{h}$ is skew symmetric, it is appropriate to set

$$
\phi^{* \hbar}(n)=\bar{\phi}(-n),
$$

which we will just denote by $\phi^{*}$, since here it does not depend on $\hbar$. The functional $\tau$, defined by $\tau(\phi)=\phi(0)$, is a trace on $S\left(Z^{d}\right)$ for $*_{h}$, and a rapid calculation shows that

$$
\tau\left(\phi *_{h} \psi^{*}\right)=\langle\phi, \psi\rangle,
$$

where the right-hand side is the usual inner-product on $L^{2}\left(Z^{d}\right)$, independent of $\hbar$. For each $\hbar$ we let $\pi_{\hbar}$ be the left regular representation of $S\left(Z^{d}\right)$ on $L^{2}\left(Z^{d}\right)$ via $*_{h}$. One verifies easily that each $\pi_{\hbar}$ is faithful on $S\left(Z^{d}\right)$. We then set

$$
\|\phi\|_{\hbar}=\left\|\pi_{\hbar}(\phi)\right\| \text {. }
$$

It is easily seen that the corresponding completed algebras, $A_{\hbar \theta}$, are noncommutative tori, as studied in [27] and the references given there, and that the notation $A_{\hbar \theta}$ agrees with that in [27]. We must now verify the conditions of Definition 1.1.

We begin with condition 2 , since this is the one which characterizes a deformation quantization. For any $\phi, \psi \in S\left(Z^{d}\right)$, and any $\hbar$ let

$$
\Delta_{\hbar}=\left(\phi *_{\hbar} \psi-\psi *_{\hbar} \phi\right) / i \hbar-\{\phi, \psi\} \text {. }
$$

Then a simple calculation shows that for $n \in Z^{d}$,

$$
\Delta_{\hbar}(n)=\sum_{m} \phi(m) \psi(n-m)\left[\left(\sigma_{\hbar}(m, n)-\sigma_{\hbar}(n, m)\right) / i \hbar-4 \pi \theta(m, n)\right] .
$$

Let $F_{h}(m, n)$ denote the expression inside [ ]. It is easily checked that there is a constant $K$ such that

$$
\left|\left(e^{i t}-e^{-i t}\right) / i t-2\right| \leqslant K|t|
$$

for every real number $t$. Accordingly, one finds that

$$
\left|F_{\hbar}(m, n)\right| \leqq K^{\prime}|\hbar||\theta(m, n)|^{2},
$$

where $K^{\prime}$ is $K$ times a factor of $(2 \pi)^{2}$. Furthermore, there is a constant $M$ such that

$$
|\theta(m, n)| \leqq M|m||n|,
$$

where here || denotes the Euclidean norm on $Z^{d} \subset R^{d}$. Thus

$$
\begin{aligned}
\left|\Delta_{h}(n)\right| & \leqq \sum_{m}|\phi(m)||\psi(n-m)|\left|F_{\hbar}(m, n-m)\right| \\
& \leqq \sum_{m}|\phi(m)||\psi(n-m)| \hbar K^{\prime \prime}|m|^{2}|n-m|^{2} \\
& =\hbar K^{\prime \prime}\left(|\cdot|^{2}|\phi|(\cdot)\right) *\left(|\cdot|^{2}|\psi|(\cdot)\right)(n),
\end{aligned}
$$

where $K^{\prime \prime}=K^{\prime} M^{2}$. Since $|\cdot|^{2}|\phi|(\cdot) \in S\left(Z^{d}\right)$ and similarly for $\psi$, the (ordinary) convolution term is in $L^{1}\left(Z^{d}\right)$. But it is easily seen that the $L^{1}$-norm dominates 
any of the norms \|\|$_{h}$, and so

$$
\left\|\Delta_{\hbar}\right\|_{\hbar} \leqq \hbar K^{\prime \prime}\left\||\cdot|^{2}|\phi|(\cdot)\right\|_{1}\left\||\cdot|^{2}|\psi|(\cdot)\right\|_{1} \text {. }
$$

It follows that $\left\|\Delta_{\hbar}\right\|_{\hbar}$ goes to 0 as $\hbar$ goes to 0 , so that condition 2 is verified.

We verify condition 1 by appealing to Corollary 2.7 of [28]. In that corollary it is shown that if $G$ is any amenable discrete group, and if $\Gamma$ is the compact set of all normalized two-cocyles on $G$ with values in $T$ with pointwise convergence, then the assignment $\gamma \mapsto A_{\gamma}$ for $\gamma \in \Gamma$ is a continuous field of $C^{*}$-algebras over $\Gamma$, where $A_{\gamma}$ is the group $C^{*}$-algebra of $G$ twisted by $\gamma$. In the present situation we take $G=Z^{d}$. Then the map $\hbar \mapsto \sigma_{\hbar}$ is a continuous map of $R$ into $\Gamma$, and $A_{h}=A_{\sigma_{h}}$. It follows that $\left\{A_{h}\right\}$ also is a continuous field.

We see that the non-commutative $d$-tori actually form a continuous field of $C^{*}$-algebras over the space of skew-symmetric real $d \times d$ matrices. If instead of the straight line path $\hbar \mapsto \hbar \theta$ in the space of skew-symmetric matrices we take any smooth path which is tangent to this straight line at 0 , it is easily seen that the corresponding field of $C^{*}$-algebras will be a strict deformation quantization of $T^{d}$ for the same Poisson structure. This shows the considerable non-uniqueness of strict deformation quantizations.

Finally, we consider the invariance under the action of $T^{d}$, as defined in Definition 1.2, of the above strict deformation quantization. The action of $T^{d}$ is just the usual dual group action, and so satisfies condition 1 of Definition 1.2. The fact that the elements of $S\left(Z^{d}\right)$ are $C^{\infty}$-vectors for the action (i.e. condition 2), and that these elements are in the domain of the corresponding Lie algebra action (i.e. condition 3), essentially goes back to Connes [7], and is discussed in [27] and elsewhere. It follows that our deformation quantization is indeed invariant. We summarize the results of this section as

1.3 Theorem. Let $\theta$ be a skew-symmetric matrix which defines a Poisson structure $\Lambda$ on $C^{\infty}\left(T^{d}\right)$ as above. For each real $\hbar$ let $A_{\hbar}$ denote the corresponding normed ${ }^{*}$-algebra obtained by Fourier transform and using the bicharacter $\sigma_{\hbar}$ for $\theta$ as above. Then the family $\left\{A_{\hbar}\right\}$ provides a strict deformation quantization for $C^{\infty}\left(T^{d}\right)$ in the direction of $\Lambda$, which is invariant under the evident action of $T^{d}$.

\section{Invariant Poisson Structures on Heisenberg Manifolds}

We will parametrize the Heisenberg group, $G$, by

$$
(x, y, z)=\left(\begin{array}{lll}
1 & y & z \\
0 & 1 & x \\
0 & 0 & 1
\end{array}\right),
$$

so that when it is identified with $R^{3}$ the product is given by

$$
(x, y, z)\left(x^{\prime}, y^{\prime}, z^{\prime}\right)=\left(x+x^{\prime}, y+y^{\prime}, z+z^{\prime}+y x^{\prime}\right) .
$$

For any positive integer $c$ we will let $D_{c}$ denote the discrete subgroup of $G$ consisting of those $(x, y, z)$ such that $x, y$ and $c z$ are integers. The corresponding Heisenberg manifold is $M_{c}=G / D_{c}$, on which $G$ acts on the left. 
Let $G$ be any connected Lie group and $D$ any closed subgroup, and set $M=G / D$. We wish to determine the $G$-invariant Poisson structures on $M$. It is easily seen that these correspond to the Poisson structures $\Lambda$ on $G$ which are invariant under left translation by $G$ and right translation by $D$. By the $G$-invariance of $\Lambda$, it will be determined by its value at the identity element of $G$, and so is given by an element, say $\Lambda$ again, of $\wedge^{2} L$, where $L$ is the Lie algebra of $G$. Then right translation invariance under $D$ will be equivalent to invariance of $\Lambda$ under the restriction to $D$ of the adjoint representation of $G$ on $L$, and so on $\wedge^{2} L$.

With $G$ now the Heisenberg group, take as basis for $L$ the elements

$$
X=\left(\begin{array}{lll}
0 & 0 & 0 \\
0 & 0 & 1 \\
0 & 0 & 0
\end{array}\right), \quad Y=\left(\begin{array}{lll}
0 & 1 & 0 \\
0 & 0 & 0 \\
0 & 0 & 0
\end{array}\right), \quad Z=\left(\begin{array}{lll}
0 & 0 & 1 \\
0 & 0 & 0 \\
0 & 0 & 0
\end{array}\right)
$$

so that $[Y, X]=Z$. Then any $G$-invariant $\Lambda \in \wedge^{2} L$ is of the form

$$
\Lambda=\mu X \wedge Z+v Y \wedge Z+\rho X \wedge Y
$$

for real numbers $\mu, v, \rho$. Let $S$ and $T$ denote the elements $(1,0,0)$ and $(0,1,0)$ of $D_{c}$. Then simple calculations show that

$$
\begin{array}{ll}
\operatorname{Ad}_{S}(X)=X, & \operatorname{Ad}_{S}(Y)=Y+Z, \\
\operatorname{Ad}_{T}(X)=X-Z, & \operatorname{Ad}_{T}(Y)=Y .
\end{array}
$$

From this and the fact that $Z$ is central, it is easily seen that $\Lambda$ is $D_{c}$-invariant if and only if $\rho=0$, so that

$$
\Lambda=(\mu X+v Y) \wedge Z
$$

Let us now express $\Lambda$ more explicitly in terms of the coordinates obtained as above by identifying $G$ with $R^{3}$. Let $\partial_{i}$ for $i=1,2,3$ denote the partial differentiation operators for the standard coordinates on $R^{3}$. Then straightforward calculations using the fact that $X, Y$ and $Z$ are to be invariant under left translation show that

$$
X=\partial_{1}+y \partial_{3}, \quad Y=\partial_{2}, \quad Z=\partial_{3} .
$$

It follows that

$$
\Lambda=\left(\mu \partial_{1}+v \partial_{2}\right) \wedge \partial_{3} .
$$

Because this operator has constant coefficients, it is easily checked directly that the corresponding Poisson bracket satisfies the Jacobi identity.

We can view the elements of $C^{\infty}\left(M_{c}\right)$ as elements of $C^{\infty}(G)$ which are invariant under right translation by $D_{c}$. Then the Poisson bracket on $C^{\infty}\left(M_{c}\right)$ is obtained by applying $\Lambda$ directly to such functions.

Probably the most straightforward path to strict deformation quantization of the Heisenberg manifolds proceeds as follows. Every element of $C^{\infty}\left(M_{c}\right)$, as a function on $G$, can be expressed (not uniquely) as the average over $D_{c}$ of some function in the Schwarz space $S(G) \simeq S\left(R^{3}\right)$, or even of one in $C_{c}^{\infty}(G)$. In fact, since the action of $D_{c}$ on $G$ is proper, the action of $D_{c}$ on the $C^{*}$-algebra $C_{\infty}(G)$ is proper in the sense defined in [29], where the role of the dense subalgebra can be played 
by $S(G)$ (or $C_{c}^{\infty}(G)$ ). The elements of $C^{\infty}\left(M_{c}\right)$ will thus be the "integrals" over $D_{c}$ of the elements $S(G)$, that is, $C^{\infty}\left(M_{c}\right)$ is the generalized fixed-point algebra for the action of $G$, as defined in [29]. It is natural then to try to proceed by first constructing a $D_{c}$-invariant strict deformation quantization of $S(G)$ by means of a minor modification of the Moyal product, then showing that the action of $D_{c}$ on this deformation quantization is still proper, and finally showing that the corresponding field of generalized fixed-point algebras is in fact a strict deformation quantization of $C^{\infty}\left(M_{c}\right)$. Thus the deformation quantization of $S(G)$ is a noncommutative "covering space" of the one we seek, with $D_{c}$ the group of "covering transformations." All of this can be carried out, and the relation so obtained between the quantization of $C^{\infty}\left(M_{c}\right)$ and that of $S(G)$ is quite useful in determining the structure of the quantization of $C^{\infty}\left(M_{c}\right)$, as we will see later.

However, the path outlined above becomes notationally more complicated than necessary, and so we will instead proceed by first making some reductions. First, to more conveniently handle the factor $c$, we reparametrize the Heisenberg group as

$$
\left(\begin{array}{ccc}
1 & y & z / c \\
0 & 1 & x \\
0 & 0 & 1
\end{array}\right)
$$

so that the product on $R^{3}$ becomes

$$
(x, y, z)\left(x^{\prime}, y^{\prime}, z^{\prime}\right)=\left(x+x^{\prime}, y+y^{\prime}, z+z^{\prime}+c y x^{\prime}\right),
$$

and now $D_{c}$ becomes the subgroup with integer entries. It is easily seen that the only effect this has on the invariant Poisson structures discussed earlier is to change $\mu$ and $\nu$, so this effect can be ignored. It is easily checked that for $f \in C^{\infty}(G)$ the right translate of $f$ by $(k, m, n) \in D_{c}$ is

$$
f(x+k, y+m, z+n+c k y) \text {. }
$$

If we let $E_{c}$ be the normal subgroup of $D_{c}$ consisting of elements of form $(0, m, n)$, then the quotient, $N_{c}$, of $G$ by the right action of this subgroup looks like $R \times T^{2}$. Then to obtain $M_{c}$ it suffices to take the quotient of $N_{c}$ by the action, $\rho$, of $Z$ given on functions by

$$
\left(\rho_{k} f\right)(x, \dot{y}, \dot{z})=f(x+k, \dot{y}, \dot{z}+c k \dot{y}) .
$$

We will view functions on $N_{c}$ as functions on $G \sim R^{3}$ which are invariant under the right action of $E_{c}$, and for notational simplicity we will from now on omit the dots, e.g. write $y$ instead of $\dot{y}$. Then $\Lambda$ defines a Poisson bracket on $N_{c}$ by applying $\Lambda$ directly to such functions. It is clear that $G$ acts on the left on $N_{c}$, with this action commuting with the action $\rho$.

Our path to the deformation quantization of $M_{c}$ will then more or less consist of forming a deformation quantization of $N_{c}$, then showing that the action $\rho$ on this quantization is proper and, finally, showing that the corresponding generalized fixed-point algebras form, in fact, a strict deformation quantization of $C^{\infty}\left(M_{c}\right)$.

The structure of the resulting algebras is somewhat different depending on whether or not $\mu=0$, because our parametrization of the Heisenberg group is not 
symmetric in $x$ and $y$. We will consider these two cases separately, as they each suggest different interesting generalizations.

\section{The Case $\mu=0$}

In this case $\Lambda$ only involves the second and third variables of $N_{c} \simeq R \times T^{2}$, and in those variables $\Lambda$ acts just as for the non-commutative tori discussed earlier. We generalize this situation as follows. We will replace $R$ by any Lie group $G$, and replace $Z$ by any cocompact lattice $\Gamma$ in $G$. We will replace $T^{2}$ by $T^{d}$ for some positive integer $d$, and we will take $\Lambda$ to be defined on $T^{d}$ exactly as in Sect. 1 for the non-commutative tori. Finally, we will assume that $\beta$ is a homomorphism of $\Gamma$ into $S L(d, Z)$, with corresponding action on $T^{d}$, which we require to preserve $\Lambda$. (We could allow $G L(d, Z)$, but then later formulas would be complicated by the presence of $\operatorname{det}\left(\beta_{k}\right)= \pm 1$.) As before, let $\theta$ denote the skew-adjoint real matrix which defines $\Lambda$ with respect to the standard coordinates on $T^{d}$. Then a simple calculation shows that the requirement that $\beta$ preserve $\Lambda$ is equivalent to the requirement that

$$
\beta_{k} \theta \beta_{k}^{*}=\theta
$$

for every $k \in \Gamma$, where $\beta_{k}^{*}$ denotes the transpose of $\beta_{k}$. Thus $\beta_{k}$ is "symplectic" with respect to $\theta$.

We will let $\Lambda$ also denote the corresponding Poisson structure on $G \times T^{d}$ coming from $\Lambda$ on $T^{d}$, ignoring the coordinates of $G$. And we will let $\rho$ denote the diagonal action of $\Gamma$ on $G \times T^{d}$ for $\beta$ and right translation on $G$, so that for $f$ a function on $G \times T^{d}$ we have

$$
\left(\rho_{k} f\right)(u, \dot{t})=f\left(u k, \beta_{k}^{-1}(t)\right)
$$

where $t \in R^{d}$ so that $\dot{t} \in T^{d}=(R / Z)^{d}$. (But from now on we will omit the dots for notational simplicity.) The action $\rho$ of $\Gamma$ on $G \times T^{d}$ is free and proper, and so the quotient $M=\left(G \times T^{d}\right) / \rho$ is a manifold, which is compact since $\Gamma$ is cocompact. Since $\Lambda$ on $G \times T^{d}$ is invariant under $\rho$, it defines a Poisson structure on $M$, which we also denote by $\Lambda$.

We wish to deform $C^{\infty}(M)$ in the direction of $\Lambda$. To do this we follow the path indicated earlier, of viewing $C^{\infty}(M)$ as the generalized fixed-point algebra for the action $\rho$ of $\Gamma$ on $G \times T^{d}$, and so of forming first a $\rho$-invariant deformation quantization of $C_{c}^{\infty}\left(G \times T^{d}\right)$. For this purpose we take Fourier series in the variables of $T^{d}$, to obtain $S\left(Z^{d}\right)$ with convolution, as in Sect. 1 . Thus we want to consider the $C^{\infty}$-functions on $G \times Z^{d}$ which are of compact support in $G$ and all of whose derivatives with respect to the Lie algebra of $G$ vanish at infinity in the $Z^{d}$ variables more rapidly than any polynomial grows. We will denote this collection of functions by $S_{c}\left(G \times Z^{d}\right)$, and equip it with the product

$$
(\phi * \psi)(u, n)=\sum_{m} \phi(u, m) \psi(u, n-m)
$$

for $u \in G$ and $m, n \in Z^{d}$, and with the corresponding involution. A simple calculation 
shows then that $\rho$ is given by

$$
\left(\rho_{k} \phi\right)(u, m)=\phi\left(u k, \beta_{k}^{*} m\right) .
$$

The Poisson bracket is now given, using the notation from Sect. 1, by

$$
\{\phi, \psi\}(u, n)=4 \pi \sum_{m} \phi(u, m) \psi(u, n-m) \theta(m, n) .
$$

A simple calculation shows that $\rho$ preserves the Poisson bracket in the sense that

$$
\left\{\rho_{k}(\phi), \rho_{k}(\psi)\right\}=\rho_{k}(\{\phi, \psi\}) \text {. }
$$

Then, following the steps of Sect. 1, we define

$$
\sigma_{\hbar}(m, n)=e(\hbar \theta(m, n)),
$$

and then for $\phi, \psi \in S_{c}\left(G \times Z^{d}\right)$ we define

$$
\left(\phi *_{h} \psi\right)(u, n)=\sum_{m} \phi(u, m) \psi(u, n-m) \sigma_{\hbar}(m, n) .
$$

Similarly we set

$$
\phi^{*_{h}}(u, n)=\bar{\phi}(u,-n),
$$

which we will denote just by $\phi^{*}$. We define a trace $\tau$ on $S_{c}\left(G \times Z^{d}\right)$ by

so that

$$
\tau(\phi)=\int_{G} \phi(u, 0) d u
$$

$$
\tau\left(\phi *_{h} \psi^{*}\right)=\langle\phi, \psi\rangle,
$$

the usual inner-product on $L^{2}\left(G \times Z^{d}\right)$. Then we have the evident $*$-representation, $\pi_{\hbar}$, of $S_{c}\left(G \times Z^{d}\right)$ for each $\hbar$, and the corresponding operator norm and $C^{*}$ completion. As before, one can show that this provides a strict deformation quantization for $C_{c}^{\infty}\left(G \times T^{d}\right)$. The corresponding $C^{*}$-algebras will just be $C_{\infty}(G) \otimes A_{\hbar \theta}$. We do not examine this in more detail since it is not the algebra we want. Rather, it can be considered to be a "covering space" of the algebra we want, with $\Gamma$ as the group of "covering transformations," and with the algebra we want being the generalized fixed-point algebra.

Thus what we want to determine is whether the generalized fixed-point algebra for $\rho$ is, as $\hbar$ varies, a strict deformation quantization of the algebra for $\hbar=0$, which is (the partial Fourier transform of) $C(M)$. We remark that we are in the setting of "crossed products by diagonal actions" studied in [24], except that we are also keeping track of the $C^{\infty}$-structure. Because $\rho$ does not depend on $\hbar$, it is most convenient to first go back to $C_{c}^{\infty}\left(G \times T^{d}\right)$. In view of the definition of $\rho$, the generalized fixed-point algebra here which is identified with $C^{\infty}(M)$, consists of the $F \in C^{\infty}\left(G \times T^{d}\right)$ which satisfy

$$
F\left(u k, \beta_{k}^{-1}(t)\right)=F(u, t)
$$

for all $k \in \Gamma$. Note that derivatives in $t$ of such $F$ need not satisfy this equation, since translation on $T^{d}$ does not commute with $\beta$. But each such $F$ is determined by its values in a fundamental domain, which can be chosen precompact since $\Gamma$ is cocompact. In particular, $F$ is necessarily bounded. 
For any $F \in C^{\infty}\left(G \times T^{d}\right)$ let $F^{\prime}$ denote its Fourier transform in the variables of $T^{d}$ as earlier. Then for any polynomial $P$ in $m \in Z^{d}$ and any finite product $\tilde{X}$ of elements of the Lie algebra of $G$, the function $P(m)(\tilde{X} \hat{F})(u, m)$ will be bounded for $u$ in any compact set and for all $m$. In fact, it is easily seen that this property characterizes these partial Fourier transforms of functions in $C^{\infty}\left(G \times T^{d}\right)$ from among the functions in $C^{\infty}\left(G \times Z^{d}\right)$. The invariance under $\rho$ is expressed by the same equation as earlier. Thus the generalized fixed-point algebra which we want to consider consists of the functions $\Phi \in C^{\infty}\left(G \times Z^{d}\right)$ which satisfy

1. $\Phi\left(u k, \beta_{k}^{*} m\right)=\phi(u, m)$ for all $k \in \Gamma, u \in G$, and $m \in Z^{d}$.

2. For any polynomial $P$ in $m \in Z^{d}$, and any finite product, $\tilde{X}$, of elements of the Lie algebra of $G$, the function $P(m)(\tilde{X} \Phi)(u, m)$ is uniformly bounded for $u$ in any fixed compact set and for all $m$.

We denote this space of functions by $S^{\rho}$. Note that $P(m) \Phi(u, m)$ will not usually satisfy property 1), just as earlier we saw that derivatives of $F$ in the $t$ variables do not satisfy $\rho$-invariance.

For each $\hbar$ and for all $\Phi, \Psi \in S^{\rho}$ we define the product and involution by the same formulas as before, so that

$$
\begin{aligned}
\left(\Phi *_{h} \Psi\right)(u, n) & =\sum_{m} \Phi(u, m) \Psi(u, n-m) \sigma_{\hbar}(m, n), \\
\Phi^{*}(u, n) & =\bar{\Phi}(u,-n) .
\end{aligned}
$$

It is easily checked that these are again in $S^{\rho}$. We wish to define a $*$-representation $\pi_{\hbar}$ of the resulting algebra on $L^{2}\left(G \times Z^{d}\right)$ by

$$
\left(\pi_{\hbar}(\Phi) \xi\right)(u, m)=\sum_{m} \Phi(u, m) \xi(u, n-m) \sigma_{h}(m, n),
$$

but we need to examine why the operator so defined is bounded. For this we use the evident fact that $\pi_{h}(\Phi)$ can be viewed as a field of operators on $L^{2}\left(Z^{d}\right)$ parametrized by $u \in G$, which we denote by $\pi_{h}(\Phi(u, \cdot))$. Now by hypothesis 2 in the definition of $S^{\rho}$, for any compact subset $U \subseteq G$, and any positive integer $q$, there is a constant $N$ such that

$$
|\Phi(u, m)| \leqq N\left(1+|m|^{q}\right)^{-1}
$$

for all $u \in U$ and $m \in Z^{d}$. Consequently there is a constant $N^{\prime}$ such that

$$
\|\Phi(u, \cdot)\|_{1} \leqslant N^{\prime}
$$

for all $u \in U$, where \|\|$_{1}$ is the norm for $L^{1}\left(Z^{d}\right)$. It follows that as operators on $L^{2}\left(Z^{d}\right)$

$$
\left\|\pi_{h}(\Phi(u, \cdot))\right\| \leqslant N^{\prime}
$$

for $u \in U$. We must now use condition 1 to extend this bound uniformly over $G$. For any $k \in \Gamma$ let $B_{k}$ be the unitary operator on $L^{2}\left(Z^{d}\right)$ defined by

$$
\left(B_{k} \xi\right)(m)=\xi\left(\beta_{k}^{*}(m)\right) \text {. }
$$

Then a simple calculation shows that for $\phi \in S\left(Z^{d}\right)$,

$$
\pi_{h}\left(\phi\left(\beta_{k}^{*}(\cdot)\right)\right)=B_{k} \pi_{h}(\phi) B_{k}^{-1}
$$


as operators on $L^{2}\left(Z^{d}\right)$. Thus for any $\Phi \in S^{p}$ and for fixed $u$ and $k$,

$$
\pi_{\hbar}(\Phi(u k, \cdot))=\pi_{\hbar}\left(\Phi\left(u,\left(\beta_{k}^{*}\right)^{-1}(\cdot)\right)=B_{k}^{-1} \pi_{k}(\Phi(u, \cdot)) B_{k} .\right.
$$

Since $B_{k}$ is unitary, we conclude that

$$
\left\|\pi_{\hbar}(\Phi(u k, \cdot))\right\|=\left\|\pi_{\hbar}(u, \cdot)\right\|
$$

as operators on $L^{2}\left(Z^{d}\right)$. If we now take as $U$ a compact fundamental domain for $\Gamma$ in $G$ and use the earlier estimate, we conclude that $\left\|\pi_{h}(\phi(u, \cdot))\right\|$ is uniformly bounded in $u$, so that $\pi_{\hbar}(\Phi)$ is indeed a bounded operator on $L^{2}\left(G \times Z^{d}\right)$. Accordingly, we define

$$
\|\Phi\|_{h}=\left\|\pi_{h}(\Phi)\right\|
$$

and we let $A_{\hbar}$ denote the corresponding pre- $C^{*}$-algebra.

Of course for $\hbar=0$ the algebra $A_{\hbar}$ is just (the partial Fourier transform of) $C^{\infty}(M)$. Our objective is to show that as $\hbar$ varies, the $A_{\hbar}$ provide a strict deformation quantization of $C^{\infty}(M)$ in the direction of $\Lambda$. Here the Poisson bracket on $S^{\rho}$ is given by exactly the same formula as used above for $S_{c}\left(G \times Z^{d}\right)$. We begin by verifying condition 2 of Definition 1.1. Let

$$
\Delta_{\hbar}=\left(\Phi *_{\hbar} \Psi-\Psi *_{\hbar} \Phi\right) / i \hbar-\{\Phi, \Psi\}
$$

Then, just as in Sect. 1, we find that

$$
\Delta_{\hbar}(u, n)=\sum_{m} \Phi(u, m) \Psi(u, n-m)\left[\left(\sigma_{\hbar}(m, n)-\sigma_{\hbar}(n, m)\right) / i \hbar-4 \pi \theta(m, n)\right] .
$$

As in Sect. 1, we let $F_{h}(m, n)$ denote the expression inside [ ], and, as there, we find that there is a constant, $N$, such that for any $u \in G$ and $n \in Z^{d}$ we have

$$
\left|\Delta_{h}(u, n)\right| \leqslant \hbar N\left(|\cdot|^{2}|\Phi(u, \cdot)|\right) *\left(|\cdot|^{2}|\Psi(u, \cdot)|\right)(n) .
$$

In view of property 2 of the definition of $S^{\rho}$, we find, much as in the previous paragraph, that for any compact subset $U$ of $G$ there is a constant $N^{\prime}$ such that

$$
\left\|\Delta_{h}(u, \cdot)\right\|_{\hbar} \leqslant \hbar N^{\prime}
$$

for $u \in U$, as operators on $L^{2}\left(Z^{d}\right)$. Since $\Delta \in S^{\rho}$, we can then use property 1 of the definition of $S^{\rho}$, as done in the previous paragraph, to conclude that $\left\|\Delta_{h}\right\|_{\hbar} \leqslant \hbar N^{\prime}$ as an operator on $L^{2}\left(G \times Z^{d}\right)$. It follows that $\left\|\Delta_{\hbar}\right\|_{\hbar}$ goes to 0 as $\hbar$ goes to 0 , as desired.

We now verify condition 1 of Definition 1.1 . For this we again view any $\Phi \in S^{\rho}$ as defining, for given $\hbar$, a field of operators on $L^{2}\left(Z^{d}\right)$ indexed by $u \in G$. It is easily seen from the continuity of $\Phi$ and from condition 2 of the definition of $S^{\rho}$ that $u \mapsto \Phi(u, \cdot)$ is, in fact, continuous from $G$ to $L^{1}\left(Z^{d}\right)$. Since the $L^{1}$-norm dominates the operator norms, $u \mapsto \Phi(u, \cdot)$ is continuous for any given operator norm, \|\|$_{h}$. But, given any $\varepsilon>0$ and any $\hbar_{0}$, we can apply Theorem 1.3 above, or really the part of its proof which appeals to Corollary 2.7 of [28], to conclude that for any $u \in G$ there is a neighbourhood $N_{u}$ of $\hbar_{0}$ such that

$$
\left|\|\Phi(u, \cdot)\|_{h_{0}}-\|\Phi(u, \cdot)\|_{h}\right|<\varepsilon / 3
$$


for $\hbar \in N_{u}$. Combining this with the norm continuity indicated just above, using a standard $\varepsilon / 3$ compactness argument, we find that for any compact subset $U$ of $G$ there is a neighborhood $N_{U}$ of $\hbar_{0}$ such that

$$
\text { | }\|\Phi(u, \cdot)\|_{h_{0}}-\|\Phi(u, \cdot)\|_{h} \mid<\varepsilon
$$

for all $\hbar \in N_{U}$ and all $u \in U$. If we choose $U$ to be a fundamental domain for $\Gamma$, and if we use property 1 in the definition of $S^{\rho}$ by means of the device used earlier, we obtain the above inequality uniformly over $G$, from which it follows that $\hbar \mapsto\|\Phi\|_{\hbar}$ is continuous as desired.

Having thus shown that the algebras $A_{\hat{h}}$ form a strict deformation quantization, we now examine the invariance properties. As in Sect. 1 we have the dual action of $T^{d}$ for the $Z^{d}$ variables. But a simple calculations shows that with this action an element $t$ of $T^{d}$ does not carry $S^{\rho}$ into itself unless $\beta_{k} t=t$ for all $k \in \Gamma$. We denote the subgroup of such $t$ by $\left(T^{d}\right)^{\beta}$. We also have an action of $G$ on $S^{\rho}$ coming from left translation of $G$ on itself. Putting these together, we obtain an action $\alpha$ of the Lie group $G \times\left(T^{d}\right)^{\beta}$ on $S^{\rho}$ defined by

$$
\left(\alpha_{(v, t)} \Phi\right)(u, m)=e(t \cdot m) \Phi\left(v^{-1} u, m\right)
$$

for $(v, t) \in G \times\left(T^{d}\right)^{\beta}$. Since $\Lambda$ involves only the variables of $Z^{d}$, and is of the form discussed in Sect. 1, it is preserved by the dual action of $T^{d}$, and so is also preserved by $\alpha$. For much the same reasons, $\alpha_{(v, t)}$ is a $*$-automorphism of $A_{\hbar}$ for each $\hbar$, so that condition 1 of Definition 1.2 holds. Conditions 2 and 3 are then verified in a straightforward was by combining the arguments indicated in Sect. 1 for the $Z^{d}$ variables with the usual facts about smooth vectors for the left regular representation of $G$ on itself.

We summarize the results of this section so far by:

3.1 Theorem. Let $G$ be a Lie group, and let $\Gamma$ be a cocompact lattice in $G$. Let $\beta$ be a homomorphism of $\Gamma$ into $S L(d, Z)$ with corresponding action on $T^{d}$, and let $\rho$ be the diagonal action of $\Gamma$ on $G \times T^{d}$ obtained from combining $\beta$ with the right regular representation of $G$ on itself. Let $M=\left(G \times T^{d}\right) / \rho$, which is a compact manifold. Let $\theta$ be a skew-adjoint matrix which defines a Poisson structure on $T^{d}$ for the standard coordinates of $T^{d}$. Assume that $\beta_{k} \theta \beta_{k}^{*}=\theta$ for $k \in \Gamma$, so that the corresponding Poisson structure on $G \times T^{d}$ is preserved by $\beta$, hence $\theta$ defines also a Poisson structure, $\Lambda$, on $M$. Then a strict deformation quantization for $C^{\infty}(M)$ in the direction of $\Lambda$ is obtained, as above, by taking partial Fourier transform in the $T^{d}$ variables, and then forming the normed *-algebra $A_{\hbar}$ obtained by twisting the convolution-type product for the generalized fixed-point algebra for $\rho$ by the 2-cocyle defined by $\hbar \theta$. This deformation quantization is invariant under the action $\alpha$ of $G \times\left(T^{d}\right)^{\beta}$ defined above.

We remark that this situation can easily be generalized somewhat further by replacing $\Gamma$ by any discrete group, and in the definition of $M=\left(G \times T^{d}\right) / \rho$ replacing $G$ by any manifold $N$ on which $\Gamma$ acts freely and properly on the right with compact quotient, and on which $G$ acts on the left, the action of $G$ commuting with that of $\Gamma$.

For the purpose of obtaining structural information about the algebras $A_{h}$ just constructed, it is useful that, as generalized fixed-point algebras, they are 
closely related to the corresponding crossed-product algebras. This is the case because we are essentially in the situation discussed by Raeburn and Williams in Sect. 2 of [24]. The only difference is that we have been working with $C^{\infty}$ functions, whereas they work with continuous functions. This difference has no effect on the conclusions that are drawn in [24], and essentially no effect on the proofs. Thus we will simply state here the consequences for the present situation, without proof. (One could instead apply the slightly more complicated theory of [29].)

Given $\theta$ and $\hbar$, we will let $B_{\hbar}$ denote $S_{c}\left(G \times Z^{d}\right)$ with the product $*_{h}$ and corresponding involution and norm, as discussed near the beginning of this section. Since $\Lambda$ only involves the $Z^{d}$ variables, $B_{h}$ is a dense $*$-subalgebra of $C_{\infty}(G) \otimes C_{h}$, where $C_{\hbar}$ is the corresponding non-commutative torus. Furthermore, the action $\rho$ on $B_{\hbar}$ defined near the beginning of this section extends in an evident way to $C_{\infty}(G) \otimes C_{h}$, as a diagonal action of $\beta$, extended to $C_{h}$, with the right action of $G$ on itself. The generalized fixed point algebra for this extended action is easily seen to contain $A_{\hbar}$ as a dense *-subalgebra. Then from Theorem 2.2 of [24] we immediately obtain:

3.2 Proposition. The completion, $\bar{A}_{\hbar}$, of the algebra $A_{\hbar}$ described above is strongly Morita equivalent to the crossed product algebra $\left(C_{\infty}(G) \otimes C_{h}\right) \times{ }_{\rho} \Gamma$.

We now specialize the results of this section back to Heisenberg manifolds, with $\Lambda=-\pi v \partial_{2} \wedge \partial_{3}$. The homomorphism $\beta$ is then given by

$$
\beta_{k}=\left(\begin{array}{cc}
1 & 0 \\
-c k & 1
\end{array}\right),
$$

and so the algebra $A_{\hbar}$ consists of functions $\Phi$ on $R \times Z^{2}$ which satisfy

$$
\Phi(u+k, m-c k n, n)=\Phi(u, m, n)
$$

for all $u \in R$ and $k, m, n \in Z$. The cocycle defining the product on $A_{\hbar}$ is defined by

$$
\sigma_{\hbar}((m, n),(p, q))=e((1 / 2) \hbar v(m q-n p))
$$

for $(m, n),(p, q) \in Z^{2}$. The algebra $B_{h}$ consists of the functions in $S_{c}\left(R \times Z^{2}\right)$ with product and involution defined by the same formulas as for $A_{\hbar}$, and with action $\rho$ of $Z$ defined by

$$
\left(\rho_{k}(\phi)\right)(u, m, n)=\phi(u+k, m-c k n, n) .
$$

Then by the results of Raeburn and Williams, $A_{\hbar}$ is strongly Morita equivalent to $B_{h} \times{ }_{\rho} Z$. Thus one way to obtain properties of $A_{h}$ is to study this crossed product.

\section{The Case $\mu \neq 0$ for the Covering Space}

In the case $\mu \neq 0$ we do not have available the convenient splitting of $R$ from $T^{2}$ which we used in the previous section. Thus we must employ a different approach, which is of some interest because it leads to a generalization which works any time we have an action of $R^{d}$ on a manifold. In this section we will not yet take into account the action $\rho$ of $Z$ described in Sect. 2, and so we obtain a deformation quantization which can be considered to be a "covering space" of the one we actually seek, which is treated in the next section. We remark that in this section we do not need to insist that $\mu \neq 0$, but only that one of $\mu$ and $v$ be non-zero. 
We recall from Sect. 2 that we consider $R \times T^{2}$ with the Poisson structure given by

$$
\Lambda=-\pi^{-1}\left(\mu \partial_{1}+v \partial_{2}\right) \wedge \partial_{3}
$$

To obtain a deformation quantization we now take the Fourier transform in all three variables. We obtain $S\left(R \times Z^{2}\right)$ with convolution, and with Poisson bracket defined by

$$
\{\phi, \psi\}(r, m, n)=4 \pi \int \sum_{p, q}[(\mu s+v p) n-q(\mu r+v m)] \phi(s, p, q) \psi(r-s, m-p, n-q) d s .
$$

Just as for the non-commutative tori, we then define a skew-bicharacter $\sigma_{h}$ on $R \times Z^{2}$ by

$$
\sigma_{h}((r, m, n),(s, p, q))=e(\hbar(v(p n-q m)+\mu(s n-q r))) .
$$

Then for $\phi, \psi \in S\left(R \times Z^{2}\right)$ and $u \in R \times Z^{2}$ we define

$$
\left(\phi *_{\hbar} \psi\right)(u)=\int_{R \times Z^{2}} \phi(v) \psi(u-v) \sigma_{\hbar}(v, u-v) .
$$

This gives a strict deformation quantization of $S\left(R \times T^{2}\right)$ in the direction of $\Lambda$.

Let us now consider how the corresponding product is defined on $S\left(R \times T^{2}\right)$ itself. We will denote this product by $*$, and absorb $\hbar$ into $\mu$ and $v$ for notational simplicity. For $f, g \in S\left(R \times T^{2}\right)$ we have

$$
\begin{aligned}
(f * g)(x, y, z)= & \left(\hat{f} *_{h} \hat{g}\right)(x, y, z) \\
= & \sum_{n} e(z n) \sum_{q} \int d s \sum_{p} \hat{f}(s, p, q) e(s(x+(n-q) \mu)+p(y+(n-q) v)) \\
& \cdot \int d r \sum_{m} \hat{g}(r, m, n-q) e(r(x-q \mu)+m(y-q v)) \\
= & \sum_{n} e(z n) \sum_{q} \grave{f}(x+(n-q) \mu, y+(n-q) v, q) \grave{g}(x-q \mu, y-q v, n-q),
\end{aligned}
$$

where $\grave{f}$ denotes the partial Fourier transform in the third variable.

This last formula looks somewhat like that for the product in the crossed product algebra [22] for the action $\alpha$ of $R$ on $R \times T$ given by

$$
\alpha_{r}(x, y)=(x-r \mu, y-r v),
$$

except in a more symmetrical form. Notice that the infinitesimal generator for the corresponding action on $S(R \times T)$ is given by

$$
\lim _{r \rightarrow 0}(f(x+r \mu, y+r v)-f(x, y)) / r=\left(\left(\mu \partial_{1}+v \partial_{2}\right) f\right)(x, y) .
$$

This suggests the following generalization. We consider a manifold $M$ and a smooth action $\alpha$ of $R^{d}$ on $M$. For $n=1, \ldots, d$ let $X_{n}$ denote the vector field on $M$ corresponding to differentiation along the $n^{\text {th }}$ standard basis vector for $R^{d}$ via $\alpha$. Thus for $t=\left(r_{n}\right) \in R^{d}$ we have

$$
\lim _{h \rightarrow 0}\left(f\left(\alpha_{\hbar r}(x)\right)-f(x)\right) / \hbar=\sum r_{n}\left(X_{n} f\right)(x)
$$

for every $f \in C^{\infty}(M)$. Then on $M \times T^{d}$ let 


$$
\Lambda=-\pi^{-1} \sum X_{n} \wedge \partial_{n}
$$

where $\partial_{n}$ denotes differentiation in the $n^{\text {th }}$ direction on $T^{d}$. (With only minor modifications to the discussion which follows, we could instead treat $M \times R^{d}$, in which case Alan Weinstein has pointed out to me that $\Lambda$ is essentially the semi-direct product Poisson structure, as defined in the appendix of [32], for the action of $R^{d}$ on $M$, where $M$ is given the zero Poisson structure.) Because all the vector fields involved in the definition of $\Lambda$ commute, it is easily seen that $\Lambda$ defines a Poisson structure on $M \times T^{d}$. We seek a strict deformation quantization of $M \times T^{d}$ in the direction of $\Lambda$.

For each $f \in C_{c}^{\infty}\left(M \times T^{d}\right)$ define

$$
\grave{f}(x, p)=\int_{T^{d}} f(x, t) \bar{e}(p \cdot t) d t .
$$

Then $\grave{f} \in S_{c}\left(M \times Z^{d}\right)$, much as in Sect. 3. The Poisson bracket on $S_{c}\left(M \times Z^{d}\right)$ from $\Lambda$ will be given by

$$
\{\phi, \psi\}(x, p)=-2 i \sum_{q} \sum_{n}\left(X_{n} \phi\right)(x, q)\left(p_{n}-q_{n}\right) \psi(x, p-q)-q_{n} \phi(x, q)\left(X_{n} \psi\right)(x, p-q) .
$$

Motivated by the formula obtained earlier for the case $R \times T^{2}$, we define, for any $\hbar$, and any $\phi, \psi \in S_{c}\left(M \times Z^{d}\right)$,

$$
\begin{aligned}
\left(\phi *_{\hbar} \psi\right)(x, p) & =\sum_{q} \phi\left(\alpha_{\hbar(q-p)}(x), q\right) \psi\left(\alpha_{\hbar q}(x), p-q\right), \\
\phi^{*}(x, p) & =\bar{\phi}(x,-p) .
\end{aligned}
$$

Simple calculations show that these define a $*$-algebra, which we denote by $A_{\hbar}$. One has the evident $L^{1}$-norm on this algebra (in the $Z^{d}$ variables, for the sup-norm in the $M$ variables), and so one can then form the corresponding enveloping $C^{*}$-algebra, and then go on to study its structure. However, it is easier to remark that this $C^{*}$-algebra is isomorphic to a crossed product algebra, so that we can appeal to the well-known results about crossed products. To be specific, for any given $\hbar$ let $\gamma^{\hbar}$ be the action of $Z^{d}$ on $M$ defined by $\gamma_{p}^{\hbar}=\alpha_{-2 \hbar p}$, and let $J$ (or $J_{\hbar}$ ) be the mapping from $A_{\hbar}$ into the crossed product algebra $C^{*}\left(Z^{d}, C_{\infty}(M), \gamma^{\hbar}\right)$ defined on functions by

$$
J(\phi)(x, p)=\phi\left(\alpha_{h p}(x), p\right)
$$

Then

$$
\begin{aligned}
J\left(\phi *_{h} \psi\right)(x, p) & =\left(\phi *_{\hbar} \psi\right)\left(\alpha_{\hbar p}(x), p\right) \\
& =\sum_{q} \phi\left(\alpha_{\hbar q}(x), q\right) \psi\left(\alpha_{\hbar(q+p)}(x), p-q\right) \\
& =J(\phi) * J(\psi)(x, p) .
\end{aligned}
$$

In a similar way one verifies that $J\left(\phi^{*}\right)=J(\phi)^{*}$, and that $J$ is isometric for the $L^{1}$-norms. It is clear that the range of $J$ is dense in $C^{*}\left(Z^{d}, C_{\infty}(M), \gamma^{h}\right)$. We can then take the $C^{*}$-norm, \|\|$_{h}$, on $A_{\hbar}$ to be that from this crossed product. Since $Z^{d}$ is amenable, the norm on the crossed product is that obtained from any representation obtained by inducing [22] a faithful representation of $C_{\infty}(M)$. But one has such 
a faithful representation of $C_{\infty}(M)$ on $L^{2}(M)$ for any measure on $M$ of full spport. (Our notation will not explicitly indicate the measure.) The induced representation then acts on $L^{2}\left(M \times Z^{d}\right)$, with the action at the level of functions defined for $\xi \in L^{2}\left(M \times Z^{d}\right)$ and $\Phi \in S_{c}\left(M \times Z^{d}\right)$ by

$$
(\Phi \xi)(x, p)=\sum_{q} \Phi\left(\gamma_{p}^{h}(x), q\right) \xi(x, p-q) .
$$

Composing with $J$, we find that the corresponding representation, $\pi_{h}$, of $A_{\hbar}$ is defined by $\phi \in A_{\hbar}$ by

$$
\left(\pi_{\hat{h}}(\phi) \xi\right)(x, p)=\sum_{q} \phi\left(\alpha_{h(q-2 p)}(x), q\right) \xi(x, p-q) .
$$

We will take the norm from this representation as defining the norm on $A_{n}$. We recall from definition 3.2 of [28] that an upper semi-continuous field $\left\{B_{\omega}\right\}$ of $C^{*}$-algebras over a locally compact space $\Omega$, with maximal algebra $B$ of continuous sections, is said to be Hilbert-continuous if there exists a fixed Hilbert space $H$, and for each $\omega \in \Omega$ a faithful representation $\pi_{\omega}$ of $B_{\omega}$ on $H$ such that for any $b \in B$ the function $\omega \mapsto \pi_{\omega}\left(b_{\omega}\right)$ is continuous for the strong operator topology. This implies that $\left\{B_{\omega}\right\}$ is a continuous field.

4.2 Theorem. With $\Lambda$ and $A_{h}$ defined as above, $\left\{A_{h}\right\}$ is a strict deformation quantization of $C_{c}^{\infty}\left(M \times T^{d}\right)$ in the direction of $\Lambda$. Moreover, via the representations $\pi_{\hbar}$ defined above, $\left\{A_{\hat{h}}\right\}$ is a Hilbert-continuous field.

Proof. The proof that condition 2 holds is a somewhat simplified version of the proof of this in the upcoming Theorem 5.4, and so we do not include it here separately. The proof that condition 1 holds is based on Corollary 3.5 of [28]. For each $f \in C_{\infty}(M)$ and $p \in Z^{d}$ the function $\hbar \mapsto \gamma_{p}^{h}(f)=\alpha_{-2 \hbar p}(f)$ is clearly normcontinuous from $R$ to $C_{\infty}(M)$. Thus the main hypothesis of Corollary 3.5 of [28] is satisfied. From part c) of that corollary we conclude that $\left\{C^{*}\left(Z^{d}, C_{\infty}(M), \gamma^{h}\right)\right\}$ is a continuous field of $C^{*}$-algebras over $R$, with continuity structure given by $L^{1}\left(Z^{d}, C_{\infty}(M)\right)$, and maximal $C^{*}$-algebra of sections $C^{*}\left(Z^{d}, C_{\infty}(R \times M), \gamma\right)$, where $\gamma$ has the evident meaning. For each $\hbar$ the map $J_{\hbar}$ carries $S_{c}\left(M \times Z^{d}\right)$ onto a dense subalgebra of $L^{1}\left(Z^{d}, C_{\infty}(M)\right)$, and the norm on $A_{\hbar}$ is defined to be that coming from $C^{*}\left(Z^{d}, C_{\infty}(M), \gamma^{h}\right)$ via $J_{h}$. For any $\phi \in S_{c}\left(M \times Z^{d}\right)$ the function

$$
(x, \hbar, p) \mapsto\left(J_{\hbar} \phi\right)(x, p)=\phi\left(\alpha_{-2 \hbar p}(x), p\right)
$$

is in $C_{c}\left(Z^{d}, C_{\infty}(R \times M)\right)$ at least locally in $\hbar$, that is, we can cut it off continuously outside any interval of $\hbar$ 's of interest so that it is in $C_{c}\left(Z^{d}, C_{\infty}(R \times M)\right)$. Since by part $c$ ) of Corollary 3.5 of [28] this space consists of continuous sections for the field, it follows that $\hbar \mapsto\|\phi\|_{h}$ is continuous. Q.E.D.

We now specialize to the case discussed at the beginning of this section. In this setting we can use the usual Schwarz space.

4.3 Corollary. For $\hbar \in R$ let $A_{\hbar}$ denote $S(R \times T \times Z)$ with product and involution defined by 


$$
\begin{aligned}
\left(\phi *_{\hbar} \psi\right)(x, y, p) & =\sum_{q} \phi(x-\hbar(q-p) \mu, y-\hbar(q-p) v, q) \psi(x-\hbar q \mu, y-h q v, p-q) \\
\phi^{*}(x, y, p) & =\bar{\phi}(x, y,-p)
\end{aligned}
$$

and with norm coming from the representation on $L^{2}(R \times T \times Z)$, for Lebesgue measure, defined by

$$
(\phi \xi)(x, y, p)=\sum_{q} \phi(x-\hbar(q-2 p) \mu, y-\hbar(q-2 p) v, q) \xi(x, y, p-q) .
$$

Then, via the partial Fourier transform in the last variable, $\left\{A_{\hbar}\right\}$ is a strict deformation quantization of the manifold $R \times T^{2}$ in the direction of the Poisson structure given by

$$
\Lambda=-\pi^{-1}\left(\mu \partial_{1}+v \partial_{2}\right) \wedge \partial_{3} .
$$

\section{The Case $\boldsymbol{\mu} \neq 0$ as Generalized Fixed-Point Algebra}

In this section we take into account the action $\rho$ of $Z$ described in Sect. 2, and we obtain our desired strict deformation quantizations as generalized fixed-point algebras under $\rho$ of the deformation quantizations studied in the previous section. This approach is useful in proving the continuous field property, as well as in obtaining information about the structure of the resulting algebras.

Recall from Sect. 2 that the action $\rho$ on $R \times T^{2}$ was defined by

$$
\left(\rho_{k} f\right)(x, y, z)=f(x+k, y, z+k z y) .
$$

In keeping with the generalization made in Sect. 4, we wish to define an analogous action, $\rho$, on $M \times T^{d}$, where $M$ replaces $R \times T$, and $T^{d}$ replaces the last $T$ of $R \times T^{2}$. Accordingly we let $\beta$, replacing $(x, y) \mapsto(x+k, y)$, be a (free) proper smooth action of $Z$ on $M$ which commutes with the smooth action $\alpha$ of $R^{d}$, and is such that the orbit space, $M / \beta$, is compact. (Eventually one may want to treat actions $\beta$ by other groups than $Z$, but since matters are complicated enough for $Z$, it seems best to restrict to that case here.) As we will see later, we also need to require that $\alpha$ satisfies the following growth condition with respect to $\beta$ :

5.1 Definition. With notation as above, we will say that $\alpha$ is $\beta$-bounded if there is a precompact fundamental domain $C$ in $M$ for $\beta$, and a constant $b$, such that for any $x \in C$ and any $r \in R^{d}$ there is a $k \in Z$ such that $|k| \leqq b\|r\|$ and $\alpha_{r}(x) \in \beta_{k}(C)$.

It is clear that when $\alpha$ and $\beta$ are given on $M=R \times T$ by

$$
\begin{aligned}
& \alpha_{r}(x, y)=(x-r \mu, y-r v), \\
& \beta_{k}(x, y)=(x+k, y),
\end{aligned}
$$

then $\alpha$ is $\beta$-bounded.

Continuing to generalize the original $\rho$, we will replace the factor $c y$, which we view as a function on $T^{2}$ with values in $T$, by a function, $\eta$, on $M / \beta$ with values in $T^{d}$. Since we are viewing $T^{d}$ as $R^{d} / Z^{d}$, we will write the law of composition on $T^{d}$ as addition, and will often think of $\eta$ as a $\beta$-invariant function on $M$ with values in $R^{d}$ modulo elements of $Z^{d}$. We then want to define an action, $\rho$, of $Z$ 
on $M \times T^{d}$ by

$$
\rho_{k}(x, t)=\left(\beta^{-1}(x), t+k \eta(x)\right) .
$$

We also want $\rho$ to preserve the Poisson structure on $M \times T^{d}$ used in Sect. 4 , and to be compatible with the deformation quantization defined in Sect. 4. Thus we need to put conditions on $\eta$. For this purpose and later calculations, we will need to view $T^{d}$ as the dual group of $Z^{d}$. Since we are writing the law of composition on $T^{d}$ as addition, we will write the pairing between $p \in Z^{d}$ and $t \in T^{d}$ as $e(t \cdot p)$, so that we can view $t \cdot p$ as coming from the standard inner product on $R^{d}$, but defined only modulo elements of $Z$.

We will see that the conditions we require of $\eta$ are given by:

5.2 Definition. Let $\alpha$ and $\beta$ be comuting smooth actions of $R^{d}$ and $Z$ on a manifold $M$, with $\beta$ proper and $M / \beta$ compact. We will say that a $C^{\infty}$-function $\eta$ from the manifold $M / \beta$ to $T^{d}$ is an $\alpha$-phase if

$$
e\left(p \cdot\left(\eta\left(\alpha_{n q}(x)\right)-\eta(x)\right)\right)=e\left(q \cdot\left(\eta\left(\alpha_{\hbar p}(x)\right)-\eta(x)\right)\right)
$$

for all $p, q \in Z^{d}, \hbar \in R$ and $x \in M$.

It is clear that the function $\eta(x, y)=c y$ on $R \times T$ with values in $T$ satisfies these conditions for the specific $\alpha$ and $\beta$ given earlier.

Returning to the general case, we define an action, $\rho$, of $Z$ on $M \times T^{d}$ by the formula given earlier, so that the action on functions $F$ on $M \times T^{d}$ is given by

$$
\left(\rho_{k} F\right)(x, t)=F\left(\beta_{k}(x), t-k \eta(x)\right) .
$$

Since $\beta$ is proper, it is clear that so is $\rho$. Let $N=\left(M \times T^{d}\right) / \rho$. Since $M / \beta$ is compact, so is $N$.

On $M \times T^{d}$ we have the Poisson structure defined in Sect. 4. Let us show that $\rho$ preserves this Poisson structure. If we take the derivative in $\hbar$ at 0 of the $\alpha$-phase condition of Definition 5.2, it is easily seen that in terms of standard coordinates we find that

$$
\left(X_{n} \eta_{m}\right)(x)=\left(X_{m} \eta_{n}\right)(x)
$$

for any $x \in M$ and for $1 \leqslant m, n \leqslant d$, where, even though the values of $\eta_{m}$ are determined only up to elements of $Z$, the above derivatives are well-defined as real numbers. If we now take $F$ and $G$ in $C^{\infty}\left(M \times T^{d}\right)$ and calculate $\left\{\rho_{k} F, \rho_{k} G\right\}(x, t)$ we obtain, up to the factor $-\pi^{-1}$, the following, in which for simplicity of notation it is to be understood that derivatives of $F$ and $G$ are to be evaluated at $\left(\beta_{k}(x)\right.$, $t-k \eta(x))$ :

$$
\begin{aligned}
& \sum_{n}\left[\left(X_{n} F\right)-\sum_{m}\left(\partial_{m} F\right) k\left(X_{n} \eta_{m}\right)(x)\right]\left(\partial_{n} G\right)-\sum_{n}\left(\partial_{n} F\right)\left[\left(X_{n} G\right)-\sum_{m}\left(\partial_{m} G\right) k\left(X_{n} \eta_{m}\right)(x)\right] \\
& =\rho_{k}(\{F, G\})-k \sum_{m} \sum_{n}\left(\partial_{m} F\right)\left(\partial_{n} G\right)\left(X_{n} \eta_{m}\right)(x)-\left(\partial_{n} F\right)\left(\partial_{m} G\right)\left(X_{n} \eta_{m}\right)(x) .
\end{aligned}
$$

But the last term is zero because of the fact that $X_{n} \eta_{m}=X_{m} \eta_{n}$, as found above. Thus $\rho$ preserves the Poisson structure. We also see that, at least in part, the $\alpha$-phase condition is a global form of saying that the Poisson structure is preserved. 
It follows that $\Lambda$ determines a Poisson structure on $\left(M \times T^{d}\right) / \rho$, which we will again denote by $\Lambda$. We wish to construct a deformation quantization of $C^{\infty}\left(\left(M \times T^{d}\right) / \rho\right)$. As in Sect. 4 we first consider $C_{c}^{\infty}\left(M \times T^{d}\right)$ and take partial Fourier transforms on $T^{d}$, to obtain the space $S_{c}\left(M \times Z^{d}\right)$. Then we find that $\rho$ is defined on $S_{c}\left(M \times Z^{d}\right)$ by

$$
\left(\rho_{k} \phi\right)(x, p)=\bar{e}(k p \cdot \eta(x)) \phi\left(\beta_{k}(x), p\right) .
$$

A simple calculation, using the $\alpha$-phase condition, shows that $\rho$ is an action on the algebra $A_{\hbar}$ defined in Sect. 4, for each $\hbar$. The Definitions 5.1 and 5.2 give the properties we will need in order to show that this action is proper, even in the case of the original $\rho$. In fact, one reason for our working in this generality is that it is notationally less cumbersome than working with the original $\rho$.

Since $\beta$ is proper, it is clear that the generalized fixed-point algebra for $\rho$, if it makes sense, should be the space $S^{\rho}$ of functions defined by:

5.3. Notation. Let $S^{\rho}$ denote the space of $C^{\infty}$ functions $\Phi$ on $M \times Z^{d}$ which satisfy

a) $\Phi\left(\beta_{k}(x), p\right)=e(k p \cdot \eta(x)) \Phi(x, p)$ for each $k \in Z$.

b) For every polynomial $P$ on $Z^{d}$, and every finite product $\tilde{X}$ of smooth vector fields on $M$ the function $P(p)(\tilde{X} \Phi)(x, p)$ is bounded on $K \times Z^{d}$ for any compact subset $K$ of $M$.

Note that because $\beta$ is free and proper, there are plenty of functions in $S^{\rho}$; for any $\phi \in S_{c}\left(M \times Z^{d}\right)$ the function $\sum_{k} \rho_{k}(\phi)$ will be in $S^{\rho}$. Now given any $\hbar$ and any $\Phi, \Psi \in S^{\rho}$, we define the corresponding product and involution by the same formulas as before, so that

$$
\begin{aligned}
\left(\Phi *_{\hbar} \Psi\right)(x, p) & =\sum_{q} \Phi\left(\alpha_{\hbar(q-p)}(x), q\right) \Psi\left(\alpha_{\hbar p}(x), p-q\right), \\
\Phi^{*}(x, p) & =\bar{\Phi}(x,-p) .
\end{aligned}
$$

Then it is easily verified that these are again in $S^{\rho}$, because of the conditions imposed on $\eta$. We will denote the corresponding $*$-algebra by $D_{h}$. It might seem desirable now to try to pass to a formula for the product which is closer to that for an ordinary crossed product, much as done in Sect. 4 . But when one tries this, the image of $S^{\rho}$ depends on $\hbar$, and this obscures the situation. Thus we will not make this passage.

Since at the level of $C^{*}$-algebras we will wish to view $D_{h}$ as consisting of multipliers on $A_{\hbar}$, we need to define the norm on $D_{\hbar}$ by extending to multipliers the representation on $L^{2}\left(M \times Z^{d}\right)$ used in the previous section to define the norm on $A_{\hbar}$. Thus for $\Phi \in D_{\hbar}$ and $\xi \in L^{2}\left(M \times Z^{d}\right)$ we set

$$
\left(\pi_{\hbar}(\Phi) \xi\right)(x, p)=\sum_{q} \Phi\left(\alpha_{\hbar(q-2 p)}(x, q)\right) \xi(x, p-q) .
$$

It is easily seen that $\Phi$ defines a bounded operator on $L^{2}\left(M \times Z^{d}\right)$. In fact, because $M / \beta$ is compact, it follows from the definition of $S^{\rho}$ that we can find, for any $\Phi \in S^{\rho}$, a function $f \in L^{1}\left(Z^{d}\right)$ such that $|\Phi(x, p)| \leqq f(p)$ for all $p \in Z^{d}$ and $x \in M$, so that $\left\|\pi_{h}(\Phi)\right\| \leqq\|f\|_{1}$. We will use this observation shortly. Anyway, for $\Phi \in S^{\rho}$ we set

$$
\|\Phi\|_{\hbar}=\left\|\pi_{h}(\Phi)\right\|
$$


On $S^{\rho}$ we find that the Poisson structure $\Lambda$ is given by formula 4.1. Because we checked that $\Lambda$ is $\rho$-invariant, it is clear that if $\Phi, \Psi \in S^{\rho}$, then $\{\Phi, \Psi\} \in S^{\rho}$.

The following theorem is the main result of this paper. The term "saturated" used here is defined in Definition 1.6 of [29].

5.4 Theorem. With $\Lambda$ and $D_{\hbar}$ defined as above, $\left\{D_{\hbar}\right\}$ is a strict deformation quantization of $C^{\infty}\left(\left(M \times T^{d}\right) / \rho\right)$ in the direction of $\Lambda$. For each $\hbar$ the action $\rho$ of $Z$ on $A_{\hbar}$ defined earlier is proper, and $D_{\hbar}$ is the generalized fixed-point algebra for this action. The action $\rho$ is also saturated, and so $D_{h}$ is strongly Morita equivalent to $A_{\hbar} \times{ }_{\rho} Z$.

Proof. We verify condition 2 of Definition 1.1 first. Let $\Phi, \Psi \in S^{\rho}$, and for any $\hbar \neq 0$ let

$$
\Delta_{\hbar}=\left(\Phi *_{\hbar} \Psi-\Psi *_{\hbar} \Phi\right) / i \hbar-\{\Phi, \Psi\} .
$$

Now a change of variables in one term shows that

$$
\begin{aligned}
\left(\Phi *_{\hbar} \Psi\right)(x, p)-\left(\Psi *_{\hbar} \Phi\right)(x, p)= & \sum_{q} \Phi\left(\alpha_{\hbar(q-p)}(x), q\right) \Psi\left(\alpha_{\hbar q}(x), p-q\right) \\
& -\Phi\left(\alpha_{\hbar(p-q)}(x), q\right) \Psi\left(\alpha_{-\hbar q}(x), p-q\right),
\end{aligned}
$$

which can be rewritten as

$$
\begin{aligned}
\sum_{q}\left[\Phi\left(\alpha_{\hbar(q-p)}(x), q\right)\right. & \left.-\Phi\left(\alpha_{\hbar(p-q)}(x), q\right)\right] \Psi\left(\alpha_{\hbar q}(x), p-q\right) \\
& +\sum_{q} \Phi\left(\alpha_{\hbar(p-q)}(x), q\right)\left[\Psi\left(\alpha_{\hbar q}(x), p-q\right)-\Psi\left(\alpha_{-\hbar q}(x), p-q\right)\right] .
\end{aligned}
$$

Splitting $\{\Phi, \Psi\}$ into two corresponding terms, we can write $\Delta_{\hbar}$ as $\Delta_{\hbar}^{\prime}+\Delta_{\hbar}^{\prime \prime}$, where

$$
\begin{aligned}
\Delta_{\hbar}^{\prime}(x, p)= & -i\left[\sum _ { q } \left[\left(\Phi\left(\alpha_{\hbar(q-p)}(x), q\right)-\Phi\left(\alpha_{\hbar(p-q)}(x), q\right)\right] \Psi\left(\alpha_{\hbar q}(x), p-q\right) / \hbar\right.\right. \\
& \left.-2 \sum_{n}\left(X_{n} \Phi\right)(x, q)\left(q_{n}-p_{n}\right) \Psi(x, p-q)\right],
\end{aligned}
$$

and similarly for $\Delta_{h}^{\prime \prime}$.

It suffices to show that $\left\|\Delta_{\hbar}^{\prime}\right\|_{\hbar}$ and $\left\|\Delta_{\hbar}^{\prime \prime}\right\|_{\hbar}$ converge to 0 as $\hbar$ goes to 0 . We give the argument only for $\Delta_{h}^{\prime}$, since the argument for $\Delta_{h}^{\prime \prime}$ is similar. We only need consider small $\hbar$ 's. So choose $\varepsilon>0$ and consider only $\hbar$ 's for which $|\hbar| \leqq \varepsilon$. Let $K$ be a fixed compact subset of $M$ containing a fundamental domain $C$ for $\beta$. Fix $p$ and $q$, and let

$$
K^{\prime}=\left\{\alpha_{\hbar(p-q)}(x): x \in K,|\hbar| \leqq \varepsilon\right\},
$$

which is again compact. Let $\phi$ be a function in $C_{c}^{\infty}(M)$ which agrees with $\Phi(\cdot, q)$ on a neighborhood of $K^{\prime}$. Now $\phi$ is a $C^{\infty}$-vector for the action $\alpha$, essentially by hypothesis (see chapter 8 of [11]), and so we can form the three-term Taylor expansion in $\hbar$ of

$$
\phi\left(\alpha_{h(q-p)}(\cdot), q\right)-\phi\left(\alpha_{h(p-q)}(\cdot), q\right) .
$$

But for $|\hbar| \leqq \varepsilon$ and $x \in K$ the terms in $\phi$ agree with the corresponding terms in $\Phi$. 
Thus for each such $\hbar$ and $x$ we obtain a number, $h$, between 0 and $\hbar$ such that

$$
\begin{aligned}
& \Phi\left(\alpha_{\hbar(q-p)}(x), q\right)-\Phi\left(\alpha_{\hbar(p-q)}(x), q\right) \\
& =\hbar 2 \sum_{n}\left(q_{n}-p_{n}\right)\left(X_{n} \Phi\right)(x, q)+\hbar^{2} \sum_{n, m}\left(q_{n}-p_{n}\right)\left(q_{m}-p_{m}\right)\left(\left(X_{n} X_{m} \Phi\right)\left(\alpha_{h(q-p)}(x), q\right)\right. \\
& \left.\quad-\left(X_{n} X_{m} \Phi\right)\left(\alpha_{h(p-q)}(x), q\right)\right) .
\end{aligned}
$$

It is at this point that we must use the growth conditions imposed earlier on $\beta$ and $\eta$. If we differentiate conditon a) of Notation 5.3 using $\alpha$, in the direction of the $n^{\text {th }}$ standard basis vector of $R^{d}$, we find that for $y \in M$

$$
\left(X_{n} \Phi\right)\left(\beta_{k}(y), p\right)=e(k p \cdot \eta(y))\left(X_{n} \Phi\right)(y, p)+e(k p \cdot \eta(y)) 2 \pi i k p \cdot\left(X_{n} \eta\right)(y) \Phi(y, p),
$$

where $X_{n} \eta$ is defined either by the induced action of $\alpha$ on $M / \beta$, or equivalently by viewing $\eta$ as a function on $M$. A second differentiation, in the $m^{\text {th }}$ direction, yields

$$
\begin{aligned}
\left(X_{m} X_{n} \Phi\right)\left(\beta_{k}(y), p\right)= & e(k p \cdot \eta(y))\left(X_{m} X_{n} \Phi\right)(y, p) \\
& +e(k p \cdot \eta(y)) 2 \pi i k p \cdot\left(X_{m} \eta\right)(y)\left(X_{n} \Phi\right)(y, p) \\
& +e(k p \cdot \eta(y)) 2 \pi i k p \cdot\left(X_{n} \eta\right)(y)\left(X_{m} \Phi\right)(y, p) \\
& +e(k p \cdot \eta(y)) 2 \pi i k p \cdot\left(X_{m} X_{n} \eta\right)(x) \Phi(y, p) \\
& -4 \pi^{2} k^{2} e(k p \cdot \eta(y))\left(p \cdot\left(X_{m} \eta\right)(y)\right)\left(p \cdot\left(X_{n} \eta\right)(y)\right) \Phi(y, p) .
\end{aligned}
$$

For the fundamental domain $C$ let $b$ be a bound as in Definition 5.1. Then for given $x \in M$ and $q \in Z$, we can find a $k \in Z$ with $|k| \leqq b\|\hbar q\|$, and a $y \in C$ such that $\alpha_{h q}(x)=\beta_{k}(y)$. Consequently,

$$
\begin{aligned}
\left|\left(X_{m} X_{n} \Phi\right)\left(\alpha_{h q}(x), p\right)\right| \leqq & \left|\left(X_{m} X_{n} \Phi\right)(y, p)\right| \\
& +2 \pi b\|\hbar q\|\|p\|\left\|\left(X_{m} \eta\right)(y)\right\|\left|\left(X_{n} \Phi\right)(y, p)\right| \\
& +2 \pi b\|\hbar q\|\|p\|\left\|\left(X_{n} \eta\right)(y)\right\|\left|\left(X_{m} \Phi\right)(y, p)\right| \\
& +2 \pi b\|\hbar q\|\|p\|\left\|\left(X_{m} X_{n} \eta\right)(y)\right\||\Phi(y, p)| \\
& +4 \pi^{2} b^{2}\|\hbar q\|^{2}\|p\|^{2}\left\|\left(X_{m} \eta\right)(y)\right\|\left\|\left(X_{n} \eta\right)(y)\right\||\Phi(y, p)| .
\end{aligned}
$$

Since $M / \beta$ is compact, we can find a common bound for all the above derivatives of $\eta$. By part b) of Definition 5.3 we can find one $f_{\mathrm{mn}} \in S\left(Z^{d}\right)$ which dominates on $C$ all of the products of derivatives of $\Phi$ with the common bound, constants, and powers of $\|p\|$, in the above expression, so that

$$
\left|\left(X_{m} X_{n} \Phi\right)\left(\alpha_{h q}(x), p\right)\right| \leqq\left(1+\|\hbar q\|+\|\hbar q\|^{2}\right) f_{m n}(p)
$$

for all $p, q \in Z^{d},|\hbar| \leqq \varepsilon$, and $x \in C$. We can also find $g \in S\left(Z^{d}\right)$ such that

$$
\left|\Psi\left(\alpha_{\hbar q}(x), p\right)\right| \leqq g(p)
$$

for all $p, q, \hbar$ and $x \in C$. Now $\left|\Delta_{h}^{\prime}(x, p)\right|$ can itself be rewritten as a sum of two terms to yield 


$$
\begin{aligned}
\left|\Delta_{\hbar}^{\prime}(x, p)\right| \leqq & \mid \sum_{q}\left[\left(\Phi\left(\alpha_{\hbar(q-p)}(x), q\right)-\Phi\left(\alpha_{\hbar(p-q)}(x), q\right)\right) / \hbar\right. \\
& \left.-2 \sum_{n}\left(q_{n}-p_{n}\right)\left(X_{n} \Phi\right)(x, q)\right] \Psi\left(\alpha_{\hbar q}(x), p-q\right) \mid \\
& +2\left|\sum_{q} \sum_{n}\left(q_{n}-p_{n}\right)\left(X_{n} \Phi\right)(x, q)\left[\Psi\left(\alpha_{\hbar q}(x), p-q\right)-\Psi(x, p-q)\right]\right| .
\end{aligned}
$$

By the previous inequalities and the earlier Taylor expansion, the first of these two terms is

$$
\begin{aligned}
& \leqq \hbar \sum_{q} \sum_{n, m}\left|q_{n}-p_{n}\right|\left|q_{m}-p_{m}\right| 2\left(1+\varepsilon\|p-q\|+\varepsilon^{2}\|p-q\|^{2}\right) f_{m n}(q) g(p-q) \\
& =\hbar \sum_{m, n} \sum_{q} f_{m n}(q) h_{m n}(p-q)=\hbar \sum_{m, n}\left(f_{m n} * h_{m n}\right)(p),
\end{aligned}
$$

where $h_{\mathrm{mn}}(p-q)$ is the product of $g(p-q)$ by the term in $p-q$ appearing as coefficients. Since each $f_{m n}$ and $h_{m n}$ is in $S\left(Z^{d}\right)$, so is the sum of their convolutions. By a similar but easier argument we can dominate the second term in the splitting of $\Delta_{\hbar}^{\prime}$ by the product of $\hbar$ with an element of $S\left(Z^{d}\right)$. We can apply similar arguments to $\Delta_{\hbar}^{\prime \prime}$. Putting all this together, we find $f \in S\left(Z^{d}\right)$ such that

$$
\left|\Delta_{\hbar}(x, p)\right| \leqq \hbar f(p)
$$

for all $p$ and all $x \in C$. But $\Delta_{h} \in S^{\rho}$, and so by condition a) of Definition 5.3 this inequality holds for all $x \in M$. Then

$$
\left\|\Delta_{h}\right\|_{\hbar} \leqq \hbar\|f\|_{1},
$$

from which it is clear that $\left\|\Delta_{h}\right\|_{h} \rightarrow 0$ as $\hbar \rightarrow 0$. Thus condition 2 of Definition 1.1 has been verified.

We now verify condition 1 . For this purpose it is not necessary to keep track of the $C^{\infty}$-structure, and it is simpler not to do so. Accordingly we will from now on let $D_{\hbar}$ denote the $C^{*}$-algebra completion of the algebra $D_{h}$ used above. We need to show that $\left\{D_{h}\right\}$ is a continuous field over $R$, where as continuity structure we can, instead of $S^{\rho}$, just take the simpler space, $C^{\rho}$, of functions $\Phi$ on $M \times Z^{d}$ which are of compact support on $Z^{d}$ and satisfy condition a) of Notation 5.3.

We wish to appeal to Theorem 3.2 of [29], and so we must verify the hypotheses of that theorem. For each $\hbar$ let $A_{\hbar}$ denote the $C^{*}$-completion of the $A_{\hbar}$ of Sect. 4. Then when convenient, we can view $A_{\hbar}$ as the completion of $C_{c}\left(M \times Z^{d}\right)$ instead of $S_{c}\left(M \times Z^{d}\right)$. According to Theorem 4.2, the field $\left\{A_{h}\right\}$ is Hilbert-continuous, with representations $\sigma_{\hbar}$ of $A_{\hat{h}}$ on $L^{2}\left(M \times Z^{d}\right)$ defined by the same formula as that which defines the representation $\pi_{\hbar}$ of $D_{\hbar}$ given above. It is clear that the algebra, $A$, of continuous cross sections of $\left\{A_{\hbar}\right\}$ which vanish at infinity, has $A^{0}=$ $C_{c}\left(M \times Z^{d} \times R\right)$ as dense $*$-subalgebra, with operations defined as above except parametrized by $\hbar \in R$. We let $\pi_{\hbar}$ be the evident homomorphism of $A$ onto $A_{\hbar}$, and we let $\rho$ denote, in addition to the action on each $A_{\hbar}$, also the action on $A$ defined for $F \in A^{0}$ by

$$
\left(\rho_{k}(F)\right)(x, p, \hbar)=\bar{e}(k p \cdot \eta(x)) F\left(\beta_{k}(x), p, \hbar\right)
$$


for $k \in Z$. Then each $\pi_{\hbar}$ is equivariant for $\rho$. Thus $\rho$ is a continuous field of actions, as defined in Definition 3.1 of [28]. We wish to show that $\rho$ is in fact a continuous field of proper actions, as defined in Definition 3.1 of [29], that is, that $\rho$ as an action on $A$ is proper, as defined in Definition 1.2 [29]. As dense *-subalgebra for Definition 1.2 we take $A^{0}$ defined above. Now because $\beta$ is a proper action of $Z$ on $M$, a straightforward examination shows that for each $F, G \in A^{0}$ there is only a finite set of $k$ 's for which $F \rho_{k}(G) \neq 0$. Thus condition 1) of Definition 1.2 is satisfied.

We verify condition 2 ) of Definition 1.2 in the slightly stronger form that, for every $F \in A^{0}$ there is a $\Theta \in M(A)$ which carries $A^{0}$ into itself and is such that for all $G \in A^{0}$ we have

$$
\sum G \rho_{k}(F)=G \Theta
$$

The natural candidate for $\Theta$ is defined, as a function, by

$$
\begin{aligned}
\Theta(x, p, \hbar) & =\sum \rho_{k}(F)(x, p, \hbar), \\
& =\sum_{k} \bar{e}(k p \cdot \eta(x)) F\left(\beta_{k}(x), p, \hbar\right) .
\end{aligned}
$$

Because $F$ has compact support and $\beta$ is proper, $\Theta$ is well defined. It is clear that $\Theta(\cdot, \cdot, \hbar)$ is invariant under $\rho$ for each $\hbar$, so that $\Theta \in C_{c}\left(R, C^{\rho}\right)$. Since each element of $C^{\rho}$ defines an element of $M\left(A_{\hbar}\right)$ for any $\hbar$, with norm uniformly bounded in $\hbar$, and since $\Theta$ carries $A^{0}$ into itself, it is clear that $\Theta$ defines an element of $M\left(A^{0}\right)$. A simple calculation then shows that $\sum G \rho_{k}(F)=G \Theta$ for any $G \in A^{0}$. Thus $\rho$ does indeed satisfy all the conditions for being a proper action of $Z$ on $A$.

Because $Z$ is amenable, the full and reduced crossed products of $Z$ with each $A_{h}$ agree [22]. And we have seen in Theorem 4.2 that the field $\left\{A_{h}\right\}$ is Hilbert-continuous. Thus we have verified the hypotheses of Theorem 3.2 of [29], and can conclude that the field of generalized fixed-point algebras is continuous. But we have seen above that this field is just $\left\{D_{h}\right\}$. This concludes the proof that $\left\{D_{h}\right\}$ is a strict deformation quantization of $C^{\infty}\left(\left(M \times T^{d}\right) / \rho\right)$.

Next, we show that the action $\rho$ on $A_{h}$ is saturated. For this we use criterion 3) of Proposition 2.3 of [29], namely we show that the ideal $E$ defined there, which is the closed linear span of functions $\left\langle\phi, \psi^{*}\right\rangle_{E}(k)=\phi \rho_{k}(\psi)$ in $C_{c}\left(Z, C_{c}\left(M \times Z^{d}\right)\right)$, for $\phi, \psi \in C_{c}\left(M \times Z^{d}\right)$, is carried into itself by the dual action $\hat{\rho}$. We could instead use criterion 2), consisting of showing directly that $E$ contains an approximate identity for the crossed-product, by means of arguments similar to those in [26], but this would be more complicated. However, we should remark that the proof of criterion 3) given in [29] depends on a substantial theorem of Gootman and Lazar.

The argument which follows is simply a slightly more complicated version of the argument used in the proof of Corollary 2.4 of [29]. Let $t \in T=\hat{Z}$, and let $\phi$, $\psi \in C_{c}\left(M \times Z^{d}\right)$. It suffices to show that $\hat{\rho}_{t}\left(\left\langle\phi, \psi^{*}\right\rangle_{E}\right)$ is again in $E$. We can assume that $\phi$ and $\psi$ are each, in their second variable, supported on only one point of $Z^{d}$, since otherwise we can express each of them as a finite sum of elements of $C_{c}\left(M \times Z^{d}\right)$ which have this property. Suppose the supports are at $q$ and $p-q$ respectively. Then for all $k \in Z$,

$$
\hat{\rho}_{t}\left(\left\langle\phi, \psi^{*}\right\rangle_{E}\right)(k)=e(k t)\left\langle\phi, \psi^{*}\right\rangle_{E}(k)=e(k t)\left(\phi \rho_{k}(\psi)\right),
$$


which will only be non-zero at $p$, and evaluated there and at any $x \in M$, is

$$
=e(k t) \phi\left(\alpha_{\hbar(q-p)}(x), q\right) \bar{e}\left(k(p-q) \cdot \eta\left(\alpha_{\hbar q}(x)\right)\right) \psi\left(\beta_{k}\left(\alpha_{\hbar q}(x)\right), p-q\right) .
$$

Since $\beta$ is a free and proper action of $Z$, we can assume that all the translates of the closure of the support of $x \mapsto \psi(x, p-q)$ are disjoint, for if this is not the case, then we can express $\psi$ as a finite sum of elements of $C_{c}\left(M \times Z^{d}\right)$ which do have this property, and are all still supported at $p-q$ in the second variable. Likewise we can then assume that there is at most one $k_{0} \in Z$ such that the support of $x \mapsto \phi\left(\alpha_{\hbar(q-p)}(x), q\right)$ meets the support of $x \mapsto \psi\left(\beta k_{0}\left(\alpha_{n q}(x)\right), p-q\right)$. If there is no such $k_{0}$, then $\langle\phi, \psi\rangle_{E}=0$, and so $\hat{\rho}_{t}$ applied to it is in $E$. Otherwise, let $\theta=\bar{e}\left(k_{0} t\right) \psi$. Then for any $x \in M$ and $k \in Z$,

$$
\hat{\rho}_{t}\left(\left\langle\phi, \psi^{*}\right\rangle_{E}\right)(k, x)=e(k t)\left\langle\phi, \psi^{*}\right\rangle_{E}(k, x),
$$

which, by the computation above, is 0 if $k \neq k_{0}$, and so can be rewritten as

$$
\begin{aligned}
& =\phi\left(\alpha_{\hbar(q-p)}(x), q\right) \bar{e}\left(k(p-q) \cdot \eta\left(\alpha_{\hbar q}(x)\right)\right) e\left(k_{0} t\right) \psi\left(\beta_{k}\left(\alpha_{\hbar q}(x)\right), p-q\right) \\
& =\left\langle\phi, \theta^{*}\right\rangle_{E}(x, k),
\end{aligned}
$$

as desired.

We are now in a position to apply Corollary 1.7 of [29] to conclude that each $D_{\hbar}$ is strongly Morita equivalent to $A_{\hbar} \times \rho Z$. Q.E.D.

We now specialize the above theorem to the Heisenberg manifolds.

5.5 Theorem. For any positive integer $c$ let $S^{c}$ denote the space of $C^{\infty}$ functions $\Phi$ on $R \times T \times Z$ which satisfy

a) $\Phi(x+k, y, p)=e(c k p y) \Phi(x, y, p)$ for all $k \in Z$.

b) For every polynomial $P$ on $Z$ and every partial differential operator $\tilde{X}=\partial^{m+n} / \partial x^{m} \partial y^{n}$ on $R \times T$ the function $P(p)(\tilde{X} \Phi)(x, y, p)$ is bounded on $K \times Z$ for any compact subset $K$ of $R \times T$.

For each $\hbar \in R$ let $D_{\hbar}$ denote $S^{c}$ with product and involution defined by

$$
\begin{aligned}
(\Phi * \Psi)(x, y, p) & =\sum_{q} \Phi(x-\hbar(q-p) \mu, y-\hbar(q-p) \nu, q) \Psi(x-\hbar q \mu, y-\hbar q v, p-q), \\
\Phi^{*}(x, y, p) & =\bar{\Phi}(x, y,-p),
\end{aligned}
$$

and with norm coming from the representation on $L^{2}(R \times T \times Z)$, for Lebesgue measure, defined by

$$
(\Phi \xi)(x, y, p)=\sum \Phi(x-\hbar(q-2 p) \mu, y-\hbar(q-2 p) v, q) \xi(x, y, p-q) .
$$

Then, via the partial Fourier transform in the last variable, $\left\{D_{h}\right\}$ is a strict deformation quantization of the Heisenberg manifold $C^{\infty}\left(M_{c}\right)$ in the direction of the Poisson structure given by

$$
\Lambda=-\pi^{-1}\left(\mu \partial_{1}+v \partial_{2}\right) \wedge \partial_{3} .
$$

The norm completion of each $D_{h}$ is strongly Morita equivalent to the crossed product $A_{\hbar} \times{ }_{\rho} Z$, where $A_{\hbar}$ is the $C^{*}$-algebra defined in Corollary 4.3, with space $S(R \times T \times Z)$ 
and with product, involution and norm defined by the same formulas as above, and where $\rho$ is the saturated proper action defined by

$$
\left(\rho_{k} \phi\right)(x, y, p)=\bar{e}(c k p y) \phi(x+k, y, p) .
$$

Finally we record the formulas for the action of the Heisenberg group on the deformed algebras. It is easily verified that under the partial Fourier transform the left action of the Heisenberg group is given on $S(R \times T \times Z)$ by

$$
\left(L_{(r, s, t)} \phi\right)(x, y, p)=e(p(t+c s(x-r)) \phi(x-r, y-s, p),
$$

for $r, s, t \in R$. This action, of course, commutes with the action $\rho$ of $Z$ on $S(R \times T \times Z)$ defined early in this section. Consequently, we would expect that the same formula would give an action of the Heisenberg group on the generalized fixed-point algebra for $\rho$. It is easily checked that this action does indeed carry $S^{c}$ into itself, and that for each $\hbar$ it is a $*$-homomorphism for the product and involution on $S^{c}$ which defines $D_{h}$. So we must check how things mesh with the norm. For any $r, s, t \in R$ define a unitary operator $U_{(r, s, t)}$ on $L^{2}(R \times T \times Z)$, depending on $\hbar$, by

$$
\left(U_{(r, s, t)} \xi\right)(x, y, p)=e(p(t+c s(x+\hbar p \mu-r)) \xi(x-r, y-s, p) .
$$

Then it is easily checked that, as operators on $L^{2}(R \times T \times Z)$,

$$
U_{(r, s, t)} \Phi U_{(r, s, t)} *=L_{(r, s, t)}(\Phi) \text {. }
$$

It follows that each $L_{(r, s, t)}$ gives an automorphism of the norm closure of $D_{h}$, so that condition 1 of Definition 1.2 is satisfied. But the fact that the $\Phi$ 's are $C^{\infty}$ functions whose values in the first variable are determined by the values on $[0,1]$, together with the evident smoothness of $L_{(r, s, t)}$ and $U_{(r, s, t)}$, make it easy to verify also conditions 2 and 3 of Definition 1.2. Thus we have shown:

5.6 Proposition. The strict deformation quantization of the Heisenberg manifolds described in Theorem 5.5 is invariant for the action of the Heisenberg group.

Let $D_{h}$ now denote the completed $C^{*}$-algebra. Each $D_{h}$ is a $C^{*}$-algebra with identity element on which the Heisenberg group acts. We recall that for any action $\alpha$ of a group $G$ on a $C^{*}$-algebra $A$ with identity element, we say that $\alpha$ is ergodic if the only elements of $A$ left fixed under $\alpha$ are the scalar multiples of the identity element. Not surprisingly we have:

5.7 Proposition. The action of the Heisenberg group on each $D_{h}$ is ergodic.

Proof. Note that for $\Phi \in S^{c}$ we have $\left(L_{(0,0, t)} \Phi\right)(x, y, p)=e(p t) \Phi(x, y, p)$, so $L_{t}=L_{(0,0, t)}$ can be viewed as defining an action of $T$ rather than $R$. We can then average $L_{t}$ over $T$ to obtain a faithful conditional expectation, $E$, of $D_{\hbar}$ onto its fixed point algebra for $L_{t}$. The range of $E$ quite clearly consists of the operators corresponding to $\Phi$ 's of the form

$$
\Phi(x, y, p)=F(x, y) \delta_{0}(p)
$$

where $F$ is any continuous function on $T^{2}$, and $\delta_{0}$ is the delta-function on $Z$ at 0 . Identifying the range of $E$ with $C\left(T^{2}\right)$ in this way, we see that any fixed points for $L$ must lie in $C\left(T^{2}\right)$. But $L_{(r, s, 0)}$ carries $C\left(T^{2}\right)$ into itself, and gives there the 
evident ergodic action of $R \times T$ by translation on $C\left(T^{2}\right)$. Thus the fixed-points for $L$ on $D_{h}$ must be scalar multiples of the identity, as desired. Q.E.D.

We remark that if we compose $E$ with integration of elements of $C\left(T^{2}\right)$ over $T^{2}$, we obtain an $L$-invariant trace on $D_{h}$, which is faithful because $E$ is.

In [5] some non-ergodic actions of the Heisenberg group on non-commutative tori are constructed. It would be interesting to have some kind of classification of all ergodic actions of the Heisenberg group on $C^{*}$-algebras, much as exists for compact Abelian group [17], though the fact that the Heisenberg group is not compact will make matters more complicated.

\section{Properties of the non-Commutative Heisenberg Manifolds}

In this section we will obtain some of the more accessible properties of the deformed Heisenberg manifolds and related $C^{*}$-algebras constructed in the previous sections. We concentrate on those constructed in Sect. 5, but this will require looking at those of Sect. 4. For the specific case of Heisenberg manifolds this will include those of Sect. 3.

We begin with the general case considered in Sect. 4 coming from a smooth action $\alpha$ of $R^{d}$ on a manifold $M$ with deformed algebras $A_{\hbar}$. We will here let $A_{h}$ denote the completed $C^{*}$-algebra. As seen in Sect. $4, A_{\hbar}$ is isomorphic to the transformation group $C^{*}$-algebra $C^{*}\left(Z^{d}, C_{\infty}(M), \gamma^{h}\right)$. Thus various structural questions can be handled by the extensive methods which have been developed for handling transformation group algebras (see [15] and references given there). So, for example, Theorem 5.3 of [33] gives a precise description of the primitive ideal space of $A_{h}$ in terms of the stability subgroups for the action $\gamma^{h}$, and the closures of $\gamma^{h}$-orbits in $M$.

We turn next to the more specific algebras $A_{h}$ described in Corollary 4.3, where again we now take $C^{*}$-completions. In the previous sections, where we were concerned with actually constructing a deformation quantization, it was important that we kept the role of $\hbar$ explicit. But now, when we are concerned only with the properties of the algebras so constructed, we notice that they form a field in the two parameters $\mu$ and $v$. Thus for notational simplicity and precision, we will now absorb $\hbar$ into $\mu$ and $v$ (i.e. set $\hbar=1$ ) and write $A_{\mu, v}$ instead of $A_{\hbar}$. (The case $\hbar=0$ is then just the case $\mu=0=v$.) As discussed above, these algebras will be isomorphic to $C^{*}\left(Z, R \times T, \gamma^{\mu, v}\right)$ where now

$$
\gamma_{k}^{\mu, v}(x, y)=(x-2 k \mu, y-2 k v) .
$$

If $\mu \neq 0$ then $\gamma^{\mu, v}$ is a proper (so free) action. By a theorem of P. Green [10], the crossed product will then be isomorphic to $C\left((R \times T) / \gamma^{\mu, v}\right) \otimes K$, where $K$ denotes the algebra of compact operators.

Now it is easily checked that $(R \times T) / \gamma^{\mu, v}$ becomes identified with $T^{2}$ under the mapping

$$
j(x, y)=(x / 2 \mu, y-(v / \mu) x),
$$

as long as $\mu \neq 0$. In this way the primitive ideal space, $\operatorname{Prim}\left(A_{\mu, v}\right)$, of $A_{\mu, \nu}$ is identified 
with $T^{2}$. If we now compose with $J$, we find that any $F \in C\left(T^{2}\right)$, acting on the crossed product as a central multiplier in the evident way, is pulled back to a central multiplier $\tilde{F}(x, y) \delta_{0}(p)$ on $A_{\mu, v}$ by the same formula as that defining the product in $A_{\mu, v}$, where $\widetilde{F}=F \circ j$, and $\delta_{0}$ is the delta-function at 0 on $Z$. If we then consider how the action $\rho$ of $Z$ on $A_{\mu, v}$, defined near the beginning of Sect. 5, lifts to act on $\widetilde{F}$, and so on $F$, we find that $\left(\rho_{k} \widetilde{F}\right)(x, y, p)=0$ if $p \neq 0$, while

$$
\left(\rho_{k} \tilde{F}\right)(x, y, 0)=F((x+k) / 2 \mu, y-(v / \mu)(x-k)),
$$

independent of $c$. Thus if we let $\tilde{\rho}$ denote the action of $\rho$ on $T^{2}$ obtained from the identification of $T^{2}$ with $\operatorname{Prim}\left(A_{\mu, v}\right)$ indicated above, we find that

$$
\tilde{\rho}_{k}(x, y)=(x+k / 2 \mu, y-(v / \mu) k) .
$$

If $\mu$ is irrational, then this action is free. Furthermore, a little calculation shows that $\{1,1 / 2 \mu, v / \mu\}$ is independent over the rationals exactly if $\{1, \mu, v\}$ is, and it is well known that exactly in this case all the orbits for the action on $T^{2}$ will be dense, so that there will only be one orbit closure. This means that there will be no $\rho$-invariant ideals in $A_{\mu, v}$. Applying Theorems 3.1 and 4.6(i), (vi) of [16] and examining separately the case $\mu=0$, we obtain:

6.1 Lemma. The crossed product $A_{\mu, \nu} \times{ }_{\rho} Z$ is simple if and only if $\{1, \mu, v\}$ is independent over the rationals.

Let us now denote by $D_{\mu, v}^{c}$ the $C^{*}$-completion of the algebras which in Theorem 5.5 were denoted by $D_{h}$. As seen there, $D_{\mu, v}^{c}$ is strongly Morita equivalent to $A_{\mu, v} \times{ }_{\rho} Z$ (where $\rho$ also should be decorated with $a c$ ). But from Theorem 3.1 of [25] it follows immediately that strong Morita equivalence preserves simplicity. We thus obtain:

6.2 Theorem. With notation as above, $D_{\mu, v}^{c}$ is a simple $C^{*}$-algebra if and only if $\{1, \mu, v\}$ is independent over the rationals.

If desired, the route indicated above can also be used to describe the primitive ideal space of $D_{\mu, v}^{c}$ when it is not simple.

We remark that the twisted Heisenberg group $C^{*}$-algebras which have been studied by Packer [18-21] have some resemblance to the algebras $D_{\mu, v}^{c}$, and it is an interesting question as to how strong a relationship there is. The relation seems likely at least to be an extension of the relationship between $C^{*}(H)$, where now $H$ is the discrete Heisenberg group, and $C(G / H)$, where $G$ is the Heisenberg Lie group, which is discussed in [18-20] in connection with calculating $K$-groups. But it is not clear how to formalize this relationship just at the level of $C^{*}$-algebras.

\section{Rigidity for the $S O(3)$ Action of the Sphere}

It is well known $[4,6,14]$ that there is a symplectic form, and so Poisson structure $\Lambda$, on the 2 -sphere $S^{2}$, which is invariant under the action of $S O(3)$ (and so of $S U(2))$. We show in this section that there does not exist any strict deformation quantization of $S^{2}$ in the direction of $\Lambda$ which is invariant under the action of $S O(3)$. Here we are using the strong form of invariance defined in Sect. 1. This will leave open the interesting question of whether there exist deformation quantizations 
which need not be invariant at all, or whether such exist which are invariant in the weaker sense described in [1], as well as what the relation is with the "quantum spheres" of [23].

As we are about to see, the lack of $S O(3)$-invariant deformation has little to do with Poisson structures, but reflects the fact that one cannot modify the product on $C\left(S^{2}\right)$ in an invariant way to get a non-commutative algebra. (There do exist fairly trivial modifications which given commutative algebras.) This rigidity was suggested by the results of A. Wassermann [31] showing that $S U(2)$ does not have any ergodic actions on non-type I von Neumann algebras, and indeed our proof is basically just a small fragment of his proof of that result.

7.1 Theorem. On $C^{\infty}\left(S^{2}\right)$ with its usual action of $S O(3)$, any new product, involution, and $C^{*}$-norm for which the action of $S O(3)$ is by *-automorphisms, must be commutative.

Proof. This is essentially case (V) of the section entitled "Gap strategy" of [31]. Since the argument is fairly simple, we include a self-contained sketch of it here. It depends strongly on the fact that we know exactly how the action of $S O(3)$ on $C^{\infty}\left(S^{2}\right)$ decomposes into irreducible representations, namely that there is one irreducible representation for each odd dimension.

So suppose that we have a new product, involution and $C^{*}$-norm, denoted by ${ }^{*}$, and \|\| respectively. For each odd integer $n$ let $A_{n}$ be the subspace of $A=C^{\infty}\left(S^{2}\right)$ for the $n$-dimensional representation of $S O(3)$. It is clear that left multiplication (for the new product) on $A$ by any element of $A_{1}$ intertwines the action, and so carries such $A_{n}$ into itself and acts there as a scalar multiple of the identity operator. In particular, $\left(A_{1}\right)^{2} \subseteq A_{1}$. Since * clearly must carry each $A_{n}$ into itself, and the norm is a $C^{*}$-norm, we cannot have $\left(A_{1}\right)^{2}=0$, so $A_{1}$ is spanned by a self-adjoint idempotent, say $e$. Then $e$ must act on each $A_{n}$ on the left as either 0 or 1 , and the same on the right because of $*$. In particular, $e$ is central.

Let $[$,$] denote the commutator for the new product. As a map from A_{3} \otimes A_{3}$ into $A$ it intertwines the diagonal action of $S O(3)$ on $A_{3} \otimes A_{3}$ with the action of $S O(3)$ on $A$. Thus $\left[A_{3}, A_{3}\right]$ must be an $S O(3)$ invariant subspace of $A$. But the map into $A$ clearly factors through the skew tensor product $A_{3} \wedge A_{3}$, which is known to carry exactly the three-dimensional irreducible representation of $S O(3)$. Thus $\left[A_{3}, A_{3}\right]$ must be either 0 or $A_{3}$. We wish to show that it must be 0 .

Suppose that $\left[A_{3}, A_{3}\right]$ were $A_{3}$. Choose a maximal torus in $S O(3)$, and corresponding weight vectors $a_{1}, a_{0}, a_{-1}$ in $A_{3}$. By adjusting by scalar multiples, we can arrange that $\left[a_{0}, a_{1}\right]=2 a_{1},\left[a_{0}, a_{-1}\right]=-2 a_{-1},\left[a_{1}, a_{-1}\right]=a_{0}$. Let $B$ be the *-subalgebra of $A$ generated by $A_{3}$. Then $B$ is $S O(3)$-invariant, and so must be a direct sum of certain of the $A_{n}$ 's. For any $k$ let $B_{k}$ denote the linear span of products of $k$ or less elements of $A_{3}$, so $B_{k+1}=B_{k}+B_{k} A_{3}$. By considering the well known decomposition for inner tensor products of representations of $S O(3)$, it is easily seen by induction that $B_{k}$ is contained in $A_{1} \oplus \cdots \oplus A_{2 k+1}$. Suppose that one actually had equality here for each $k$. For any $k$ let $c$ be a highest weight vector in $A_{2 k+1}$. Then $c$ would be a product of $k$ elements selected from $\left\{a_{0}, a_{1}, a_{-1}\right\}$, and so, considering the weights involved, it must be $\left(a_{1}\right)^{k}$ up to a scalar multiple. We thus find that $\left(a_{1}\right)^{k} \neq 0$ for all $k$. But by Leibnitz's rule,

$$
\left[a_{0},\left(a_{1}\right)^{k}\right]=2 k\left(a_{1}\right)^{k} \text {. }
$$


Thus taking commutators with $a_{0}$ would be an unbounded operator in the $C^{*}$ completion of $A$. It follows that we must have $B_{k+1}=B_{k}$ for some $k$, so that $B=B_{k}$ and $B$ is finite dimensional. Thus we find that there is some $n$ such that $C=A_{1} \oplus \cdots \oplus A_{n}$ is a subalgebra of $A$. Now $C$ is a finite-dimensional $C^{*}$-algebra, and so a direct sum of full matrix algebras. But $S O(3)$ has an action on $C$, and for this action the trivial representation has multiplicity 1 . It follows from this that $C$ is exactly one full matrix algebra. Let $C^{\prime}$ denote the commutant of $C$ in $A$. Then $S O(3)$ carries $C^{\prime}$ into itself, and also $A \cong C \otimes C^{\prime}$, with this decomposition respecting the action. But if $W$ is the subspace in $C^{\prime}$ for some irreducible representation of $S O(3)$, then $A_{1} \otimes W$ and $A_{3} \otimes W$ will both contain subrepresentations equivalent to $W$, contradicting the fact that irreducible representations of $S O(3)$ occur in $A$ with multiplicity 1 . Consequently we cannot have $\left[A_{3}, A_{3}\right]=A_{3}$.

Thus the only possibility left is that $\left[A_{3}, A_{3}\right]=0$. Let $B$ and $B_{k}$ be defined as above. If $B_{k+1}=B_{k}$ for some $k$, then $B$ is a finite dimensional commutative $C^{*}$ algebra on which $S O(3)$ acts. But $S O(3)$ is connected, and so its action on the primitive ideal space of $B$ would be trivial, and so its action on $C$ would be trivial, contradicting the fact that the trivial representation occurs with multiplicity 1 . Thus we conclude that $B_{k}=A_{1} \oplus \cdots \oplus A_{2 k+1}$ for all $k$, so that $B=A$, and $A$ is commutative. Q.E.D.

We remark that there are several articles in the literature about invariant deformation quantization for the sphere $[3,6,14]$. But in these articles the algebra of functions involved consists only of polynomials, and the deformations are in terms of formal power series.

It seems very likely that the results of Wassermann [31] can be used to show that invariant deformations do not exist for invariant Poisson structures on other homogeneous spaces of $S U(2)$, especially for the action of $S U(2)$ on itself.

If we pass to $S U(3)$, it is not difficult to see that if one chooses a maximal torus $T^{2}$ in $S U(3)$ and chooses a Poisson structure for $T^{2}$ as in Sect. 1, then this Poisson structure can be extended to all of $S U(3)$, and that the deformations of $T^{2}$ described in Sect. 1 can be induced to $S U(3)$ to give invariant strict deformation quantizations of $S U(3)$ acting on itself. But it is not at all clear whether any less trivial kinds of deformation quantizations can be constructed.

Acknowledgement. I would like to express here my warm thanks to Alan Weinstein, not only for having pointed out to me that non-commutative tori were examples of deformation quantization, but also for some very helpful discussions, and for showing me many articles on the subject of deformation quantization.

\section{References}

1. Arnal, D.: *-products and representations of nilpotent groups. Pacific J. Math. 114, 285-308 (1984)

2. Basart, H., Lichnerowicz, A.: Conformal symplectic geometry, deformations, rigidity and geometrical (KMS) conditions. Lett. Math. Phys. 10, 167-177 (1985)

3. Bayen, F., Flato, M., Fronsdal, C., Lichnerowicz, A., Sternheimer, D.: Deformation theory and quantization, I, II. Ann. Phys. 110, 61-110, 111-151 (1978)

4. Bayen, F., Fronsdal, C.: Quantization on the sphere. J. Math. Phys. 22, 1345-1349 (1981)

5. Bratteli, O., Elliott, G. A., Goodman, F. M., Jorgensen, P. E. T.: Smooth Lie group actions on non-commutative tori, preprint 
6. Cahen, M., Gutt, S.: Non localité d'une deformation symplectique sur la sphere $S^{2}$. Bull. Soc. Math. Belg. 36, 207-214 (1984)

7. Connes, A.: $C^{*}$-algèbres et géometrie differentielle. C. R. Acad. Sci. Paris 290, 599-604 (1980)

8. —,: Non-commutative differential geometry. Pub. I.H.E.S. 62, 257-360 (1986)

9. Dixmier, J.: Les $C^{*}$-algèbres et leurs représentations, $2^{\text {nd }}$ ed., Paris: Gauthier-Villars 1969

10. Green, P.: $\mathrm{C}^{*}$-algebras of transformation groups with smooth orbit space. Pacific J. Math. 72 , 71-97 (1977)

11. Jorgensen, P. E. T., Moore, R. T.: Operator commutation relations. Dordrecht: D. Reidel 1984

12. Lichnerowicz, A.: In: Deformations and quantization, geometry and physics. Modugno M. (ed.). pp. 103-116. Bologna: Pilagora Editrice Bologna 1983.

13. Moreno, C.: Invariant star products and representations of compact semisimple Lie groups. Lett. Math. Phys. 12, 217-229 (1986)

14. Moreno, C., Ortega-Navarro, P.: *-Products on $D^{1}(C), S^{2}$ and related spectral analysis. Lett. Math. Phys. 7, 181-193 (1983)

15. Muhly, P. S., Williams, D. P.: Transformation group $C^{*}$-algebras with continuous trace, II. J. Operator. Theory 11, 109-124 (1984)

16. Olesen, D., Pedersen, G. K.: Applications of the Connes spectrum to $C^{*}$-dynamical systems, II. J. Funct. Anal. 36, 18-32 (1980)

17. Olesen, D., Pedersen, G. K., Takesaki, M.: Ergodic actions of compact Abelian groups. J. Operator Theory 3, 237-269 (1980)

18. Packer, J. A.: $C^{*}$-algebras corresponding to projective representations of discrete Heisenberg groups. J. Operator Theory 18, 42-66 (1987)

19. - : Strong Morita equivalence for Heisenberg $C^{*}$-algebras and the positive cones of their $K_{0}$-groups. Canadian J. Math. (to appear)

20. -, Twisted group $C^{*}$-algebras corresponding to nilpotent discrete groups. Math. Scand. (to appear)

21. Packer, J. A., Raeburn, I.: The structure of twisted group $C^{*}$-algebras, preliminary version

22. Pedersen, G. K.: $C^{*}$-algebras and their automorphism groups. Lond. Math. Soc. Monographs vol. 14, London: Academic Press 1979

23. Podles, P.: Quantum spheres. Lett. Math. Phys. 14, 193-202 (1987)

24. Raeburn, I., Williams, D. P.: Pull-backs of $C^{*}$-algebras and crossed products by certain diagonal actions. Trans. A.M.S. 287, 755-777 (1985)

25. Rieffel, M. A.: Unitary representations of group extensions; an algebraic approach to the theory of Mackey and Blattner. Stud. Anal. Adv. Math. [Suppl.] Ser. 4, 43-82 (1979)

26. -, Applications of strong Morita equivalence to transformation group $C^{*}$-algebras. Operator Algebras Appl. Kadison, R. V.: (ed.). pp. 299-310. Proc. Symp. Pure Math. vol. 38, Providence, RI: American Mathematical Society 1982

27. - , Projective modules over higher dimensional non-commutative tori. Canadian J. Math. 40, 257-338 (1988)

28. - : Continuous fields of $C^{*}$-algebras from group cocycles and actions, Math. Ann. (to appear)

29. - : Proper actions of groups on $C^{*}$-algebras, preprint

30. Vey, J.: Déformation du crochet de Poisson sur une variété symplectique. Commun. Math. Helv. 50, 421-454 (1975)

31. Wassermann, A. J.: Ergodic actions of compact groups on operator algebras, III: Classification for SU(2). Invent. Math. 93, 309-354 (1988)

32. Weinstein, A.: Poisson geometry of the principal series and nonlinearizable structures. J. Diff. Geom. 25, 55-73 (1987)

33. Williams, D. P.: The topology of the primitive ideal space of transformation group $C^{*}$-algebras and C. C. R. transformation group $C^{*}$-algebras. Trans. A.M.S. 266, 335-359 (1981)

34. Woronowicz, S. L.: Twisted $S U(2)$ group. An example of a non-commutative differential calculus. Pub. R.I.M.S. Kyoto University 23, 117-181 (1987)

35. —-, Compact matrix pseudogroups. Commun. Math. Phys. 111, 613-665 (1987)

36. - : Tannaka-Krein duality for compact matrix pseudogroups. Twisted $S U(N)$ groups, Invent. Math. 93, 35-76 (1988)

Communicated by A. Connes

Received July 5, 1988 